\title{
Zur Bestimmung des Drehpunktes im Auge.
}

\section{Von}

Dr. Leopold Weiss.

Die Verschiebung des Reflexbildes der Cornea bei Bewegungen des Auges wurde von Junge*) und später von Donders**) zur Bestimmung der Lage des Drehpunktes benutzt. Junge bestimmte zu diesem Zweck die Verschiebung, indem er dieselbe Flamme sowohl bei parallelen Gesichtslinien als auch bei einer gewissen Convergenz dieser auf beiden Hornhäuten spiegeln liess und den gegenseitigen Abstand der Spiegelbilder in den beiden genannten Stellungen der Augen mass. Um zu dieser Messung das Ophthalmometer benutzen zu können, mussten die Bilder durch Reflexion nahe zu einander gebracht werden, wozu er sich eines Sextanten bediente ***). Donders mass die Verschiebung des Cornealbildes eines Anges, die bei der Drehung des Auges um einen bestimmten Winkel erfolgt.

*) Helm holtz, phys. Optik, p. 458.

**) Donder's, die Anomal. der Refract. u. Accommod. S. 156. u. Donders u. Doijer, Archiv f. d. holl. Beiträge, Bd. III, S. 561. ***) Donders u. Doijer l. c. 
Ueber das diesen Bestimmungen zu Grunde liegende Princip sagt Donders an erwähnter Stelle:

"Wenn das Bewegungscentrum des Auges mit dem Krümmungsmittelpunkt einer sphärischen Hornhaut zusammenfallen würde, so müsste ein in der Achse dieser: Hornhaut reflectirtes Bild bei einer Bewegung des Auges seinen Ort in keiner Weise ändern. Wenn im Gegentheil, wie zu erwarten war, das Bewegungscentrum hinter dem Krümmungsmittelpunkt liegell sollte, dann müsste bei einer Wendung des Auges das reflectirte Bild in derselben Richtung seinen Ort verändern und diese Ortsveränderung ist, wie eine einfache Construction zeigt, gleich dem Sinus des Bewegungswinkels, beschrieben aus dem Bewegungscentrum mit einem Radius, gleich der Entfernung zwischen Bewegungscentrum und Krümmungsmittelpunkt."

Betrachten wir die hier in Rede stehenden Verhältnisse etwas näher. Die Hornhant sei als sphärische Fläche angenommen mit einem Krümmungsradius von $8 \mathrm{Mm}$., der Drehpunkt liege 13,0 Mm. hinter dem Hornhautscheitel. In grossem Abstande vor dem Auge befinde sich in der verlängerten Hornhautaxe ein Licht, so werden die von diesem ausgehenden Strahlen an der spiegelnden Fläche der Cornea eine solche Reflexion erleiden, als kämen sie von einem Punkt, der um den halben Krümmungsradius hinter dem Hornhautscheitel liegt.

Wird jetzt eine seitliche Wendung des Auges ausgeführt, so wird hierbei der Krümmungsmittelpunkt, als vor dem Drehpunkt gelegen, auf einem Kreisbogen sich bewegen, der mit dem Abstand vom Krümmungsmittelpunkt und Drehpunkt als Radius beschrieben ist, und nach ausgeführter Bewegung sich in $c^{d}$ befinden, wenn er bei der Primärstellung sich in $\mathrm{c}$ befand.

Umstehende Figur stellt die erwähnten Verhältnisse 
im Horizontalschnitt dar. D ist der Drehpunkt, c der Krümmungsmittelpunkt, $\mathrm{m}$ der Scheitel 'der Cornea. Nach ausgeführter Drehung liegt $c$ in $c^{\prime}, m$ in $\mathrm{m}^{\prime}$, das Spiegelbild 1 in $1^{\prime}$. L ist das weit vor dem Auge befindliche Licht, dicht dahinter in o das Auge des Beobachters. Die Lage des Spiegelbildes bei der nunmehrigen Stellung der Hornhaut ergiebt sich leicht. Ein Strahl, der von $\mathrm{L}$ ausgeht und gegen den jetzt in $\mathrm{c}^{t}$ befindlichen Krümmungsmittelpunkt zielt, wird nach $\mathrm{L}$ zurückgeworfen und zwar derart, als ob er vọn einem Punkt

Fig. 1.

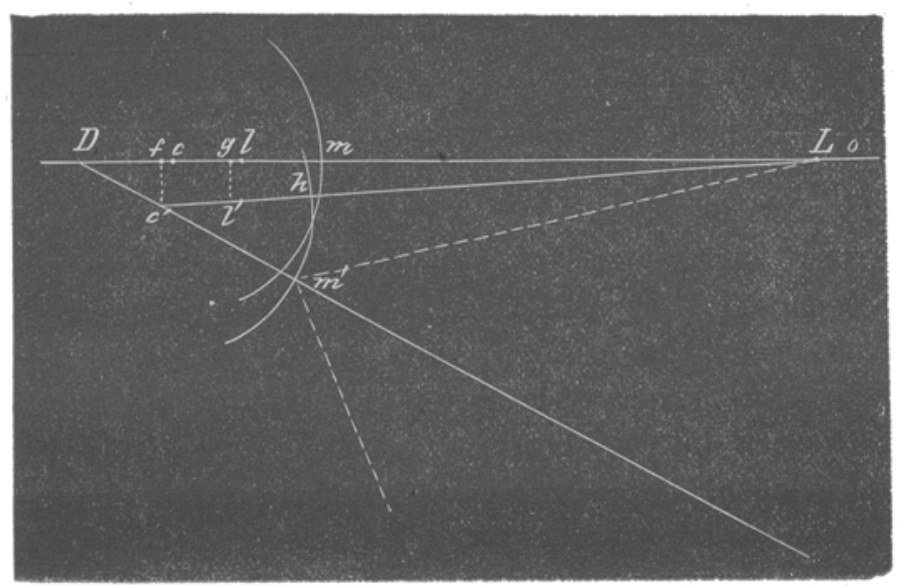

]$^{1}$ käme, der um den halben Krümmungsradius hinter der spiegelnden Fläche liegt.

Das Spiegelbild, das bei der ersten Stellung des Auges (vor der Bewegung) in 1 lag, hat sich also nach $l^{\prime}$ bewegt. Für das in o befindliche Auge des Beobachters erscheint die Grösse der Verschiebung gleich $\mathrm{gl}$ l'. g l' kann aber gleich $f c^{\prime}$ gesetzt werden, wenn das leuch- 
tende Object sehr weit vor dem Auge steht: $)-\frac{f c^{\prime}}{D c^{6}}$ ist aber $=$ dem Sinus des Drehungswinkels.

Angenommen, das Auge sei um einen Winkel von $20^{\circ}$ gedreht worden, so beträgt nach dem Vorausgehenden bei obigen Zahlen- die Verschiebung des Reflexbildes 1,7101 Mm., da

$$
\frac{f c^{\prime}}{5,0}=\sin 20^{\circ} \text { ist. }
$$

Spätere Betrachtungen, die daran anknüpfen, dass die Cornea keine sphärische Fläche ist, sondern ein Stück eines Ellipsoids darstellt - werden darthun, welche Bedeutung es für die Grösse der Verschiebung des Reflexbildes hat, welcher Punkt der Spiegeloberfiäche Licht in das Auge eines Beobachters zurückwirft, welches sich nahe hinter dem leuchtenden object befindet.

Es dürfte daher zweckmässig sein, sich hier klar zu machen, welcher Punkt der einen Convexspiegel darstellenden und als sphärisch angenommenen Hornhaut wirft im gegebenen Fall Licht, welches von $\mathrm{L}$ ausgeht, nach $L$, resp. in das dicht bei $L$ befindliche Auge des Beobachters, nachdem das Auge eine Bewegung um $20^{\circ}$ ausgeführt hat? und wie weit liegt dieser spiegelnde Punkt vom Scheitel entfernt?

Offenbar kann dies nur der Punkt $h$ sein, dessen Lage dadurch bestimmt ist, dass er auf der Verbindungslinie liegt von dem leuchtenden object und dem nunmehr (nach ausgeführter Drehung) in $c^{\prime}$ liegenden Krümmungsmittelpunkt. Alle anderen Punkte werfen kein Licht, das von $L$ ausgehend sie trifft, nack $L$ zurück, wie dies eine einfache Construction zeigt, wenn man von $\mathrm{L}$

*) Nebenbei sei bemerkt, dass, wenn das leuchtende Object sich nur $500 \mathrm{Mm}$. vor dem Auge befindet, g l' um eine mit dem Ophthalmometer messbare Grösse kleiner ist als $f c^{\prime}$. 
Strahlen an beliebige Punkte der Oberfläche z. B. an $\mathrm{m}^{\prime}$ legt und den Einfalls- resp. Reflexionswinkel construirt.

Bei der Anfangsstellung spiegelte cin Punkt m, bei der Stellung nach ausgeführter Drehung um einen bestimmten Winkel, der Punkt $h$. Wie weit liegt $h$ von dem nunmehr in $\mathrm{m}^{\prime}$ liegenden Scheitelpunkt? Die Entfernung von $\mathrm{m}^{\prime}$ lässt sich am besten als Winkelgrösse ausdrücken, wobei der Scheitel des Winkels im Krümmungsmittelpunkt $c^{\prime}$ liegt. Wie gross ist nun $h c^{\prime} m^{\prime}$ ?

Der Voraussetzung nach befindet sich das leuchtende object weit ror dem Auge. Unter dieser Voraussetzung ist es erlaubt, den Winkel $\mathbf{h} \mathbf{c}^{\prime} \mathbf{m}^{\prime}$ gleich dem Winkel CD $c^{\prime}$ zu setzen, also gleich dem Winkel, um den das Auge gedreht worden ist, denn unter genannter Voraussetzung bilden die Strahlen DL und $c^{\prime} h \mathrm{~L}$ einen verschwindend kleinen Winkel; $c^{\prime}$ h L kann daher als parallel zu DL angenommen werden.

Nach einer Drehung um $20^{\circ}$ war, wie oben auseinandergesetzt wurde, das Reflexbild um 1,71 Mm. verschoben worden.

Kennt man daher den Drehungswinkel und die Grösse der Verschiebung des Reflexbildes, so kanu man hieraus, nach dem oben Gesagten, den Abstand des Krümmungsmittelpunktes vom Drehpunkte finden.

Die Grösse des Drehungswinkels ergiebt sich aus der Verschiebung eines Fixationszeichens, welches entweder auf einem Gradbogen bewegt werden kann, welcher aus dem Drehpunkt des Auges mit einem beliebigen Radius gezogen ist und so direct den Drehungswinkel anzeigt, - oder bei bekannter Entfernung vom Auge in einer geraden Linie versehieblich ist, welche senkrecht zur Verbindungslinie mit dem Auge gezogen ist. Die Grösse der seitlichen Verschiebung zur genannten 
Verbindungslinie giebt die Tangente des Drehungswinkels.

Die Grösse der Verschiebung des Reflexes lässt sich mit dem Ophthalmometer leicht messen. Man geht von der Stellung des Anges aus, bei welcher das Cornealreflexbild von einem Licht, welches nahe über dem Fernrohr des Ophthalmometers angebracht ist, gerade in die Mitte der Hornhant fällt. Diese Stellung lässt sich auffinden nach der von Helmholtz*) herrührenden Betrachtung, dass - wenn man durch Drehung der Platten des Ophthalmometers die Hornhaut mit Reflexbild verdoppelt und die Doppelbilder um die halbe Breite der Basis corneae verschiebt - nur dann die verdoppelten Reflexbilder mit entgegengesetzten Hornhauträndern zusammenfallen, wenn das Reflexbild in der Mitte der Hornhaut liegt. - Sieht das untersuchte Auge in das Fernrohr des Ophthalmometers, so wird im Allgemeinen die genannte Bedingung nicht erfüllt. Entsprechend dem Umstand, dass für gewöhnlich die Gesiehtslinie die Hornhaut in einem Pankt schneidet, welcher nasenwärts von Hornhautmitte resp. Hornhautscheitel ${ }^{* *}$ ) liegt, wird das Auge einen Punkt fixiren müssen, welcher mehr oder weniger weit seitlich von der Mitte des Fernrohrs liegt.

Aus der Grösse, um wie viel das Fixationszeichen seitlich verschoben werden musste, lässt sich annähernd der Winkel bestimmen, welchen Hornhautaxe und Gesichtslinie bilden $* * * *$.

*) Arch. f. Ophth. Bd. I, Ab. 2, S. 22.

**) Helmholtz hat schon gezeigt, (Arch. f. Ophth. 1, 2. S. 23) dass der Scheitel der Cornea mit der Mitte derselben als zusammenfallend angesehen werden kann. Man kann daher statt durch die Mitte der Cornea auch sagen: durch den Hornhautseheitel.

***) Helmholtz 1. c. S. 23. - Ueber den hierbei entstehenden Fehler: Mauthner, die optischen Fehler des Auges, S. 66 a. 67 , 
Befindet sich das Auge in der gewünschten Stellung, so handelt es sich jetzt darum, den Ort des Reflexbildes bei dieser Stellung des Auges (sie sei die Primärstellung genannt) zu markiren. Dies geschieht nach Donders Vorgang sehr einfach dadurch, dass - nachdem der Kopf fixirt ist, - ein vertikal gespannter Faden oder ein Haar gerade vor das Reflexbild gebracht wird. Macht das Auge jetzt eine Seitwärtsdrehung, so wird das Reflexbild im Sinn der Drehung seinen Ort ändern. Die Entfernung vom Faden za dem nunmehrigen Ort des Reflexbildes ist die Grösse der Verschiebung. Diese wird mit dem Ophthalmometer gemessen, indem man das zweite Bild des Fadens mit dem ersten Reflexbild zusammenfallen lässt*).

Bezüglich der Genauigkẹit, mit der sich diese Entfernung messen lässt, sagt Donders (l. c.) „Indem man abwechselnd bald nach dem einen, bald nach dem anderen zweier Zeichen sehen liess, so konnte die erforderte Entfernung der Doppelbilder fast absolut genau gemessen werden. Die Messung war genau, wenn bei rasch $a b$ wechselnder Fixation der beiden Zeichen das eine Bild der Flamme abwechselnd vollkommen mit den beiden Haaren zusammenfiel oder um eine gleiche Grösse abwich."

Donders glaubte hierin eine einfache Methode ge-

*) Ich nehme hier gern Gelegenheit, zu erwähnen, dass die Kenntniss dieses ebenso einfachen wie sinnreichen Verfahrens mir bei der Ausführung der Bestimmungen sehr förderlich war. Meine ursprüngliche Absicht war, die Verschiebung des Reflexbildes mittelst zweier verticaler Fäden zu bestimmen, welche durch Mikrometerschrauben einzeln seitlich verschoben werden konnten. Der eine Faden sollte in der Primärstellung vor das Reflexbild gebracht werden, der andere vor dasselbe nach ansgeführter Drehung. An geeigneter Theilung sollte die Differenz abgelesen werden. 
funden zu haben, die Lage des Drehpunktes zu bestimmen; aber eine Schwierigkeit stellte sich derartigen Bestimmungen in den Weg. Die Cornea ist keine Kugelfläche, sondern stellt einen Theil eines Ellipsoids dar. Konnte die Excentricität der Ellipse, welche man erhält, wenn man einen Horizontalschnitt durch die Cornea legt, einen solchen Einfluss auf die Grösse der Verschiebung des Reflexbildes haben, dass dadurch ein merklicher Fehler entsteht?

Wie Donders angiebt, berechnete van Rees diesen Einfluss und fand, dass derselbe 2 , selbst $3,6 \mathrm{Mm}$. betragen könne.

Es müsste daher jedesmal die Ellipticität bestimmt werden, was um so nöthiger erscheint, als die Drehungen des Auges ziemlich gross sein müssen, wenn man nicht allzu kleine Verschiebungen des Reflexbildes erhalten und messen will, bei deren Messung alsdann ein selbst kleiner Fehler störend ins Gewicht fallen würde. Mit einer grossen Drehung wird aber auch ein weit von dem Hornhautscheitel gelegener Punkt zur Spiegelung kommen und aus den Eigenschaften der Ellipse geht hervor, dass der Krümmungsradius eines Punktes der Ellipse wächst mit dem Abstand des Punktes von dem Scheitel*).

Der Einfluss, den die Ellipticität der Hornhaut auf die Ortsveränderung des Refiexbildes bei Bewegungen des Auges nimmt, wird hiernach am grössten sein, wenn ein Punkt der Cornea die Spiegelung vermittelt, welcher gerade am Rande liegt.

Für diesen Grenzfall lässt sich nun der Einfluss leicht ermitteln.

*) Diese Zunahme des Krümmungsradius geschieht aber dem Abstand vom Scheitel nicht proportional, worauf ich weiter unten noch zurückkommen werde. 
Wird durch den Scheitel der Cornea ein Horizontalschnitt gelegt, so stellt dieser, wie bekannt, ein Stück einer Ellipse dar, deren halbe grosse Axe annähernd 11,5, deren halbe kleine Axe 9,5 Mm. gesetzt werden kann*).

Legt man ein rechtwinkliges Coordinatensystem zu Grunde und construirt mit gegebenen Zahlen eine Ellipse derart, dass die grosse Axe der Ellipse in die X-Axe, die kleine Axe in die ÝAxe des Coordinatensystems fällt (wobei dann das Centrum der Ellipse und der Anfangspunkt des Coordinatensystems zusammenfallen und die X-Axe durch den Hornhautscheitel geht) - so stellt der dem Durchschnittspunkt mit der X-Axe nächst benachbarte Theil der Curve die Hornhaut dar. Die Grösse dieses Theiles ist dadurch bekannt, dass man die Breite der Basis corneae,kennt. Letztere sei mit dem Mittelwerth 11,8 angenommen. Damit sind alle zur Ausführung der Rechnung nöthigen Werthe gegeben.

Es soll der Fall untersucht werden, in dem ein Punkt P am Rande der Cornea spiegelt. Fragt man zunächst, was sind die Coordinaten dieses Punktes?

Wie aus der Figur 2, welche die genannten Verhältnisse in einem Horizontalschnitt wiedergiebt, ersichtlich ist, ist die Ordinate des Punktes P am Rande der Cornea gleich dem halben Durchmesser der Basis corneae oder Pf $=5,9$; die Abscisse des Punktes P Of ergiebt sich dann aus der Ellipsengleichung:

$$
\left(\frac{x}{a}\right)^{2}+\left(\frac{y}{b}\right)^{2}=1
$$

*) Als Mittel aus den an 3 Augen von Helmholtz ausgetahrten Messungen erhält man für die halbe grosse Axe 11,612 $\mathrm{Mm}$. und für die halbe kleine Axe 9,44 Mm. 
Tig, ?.

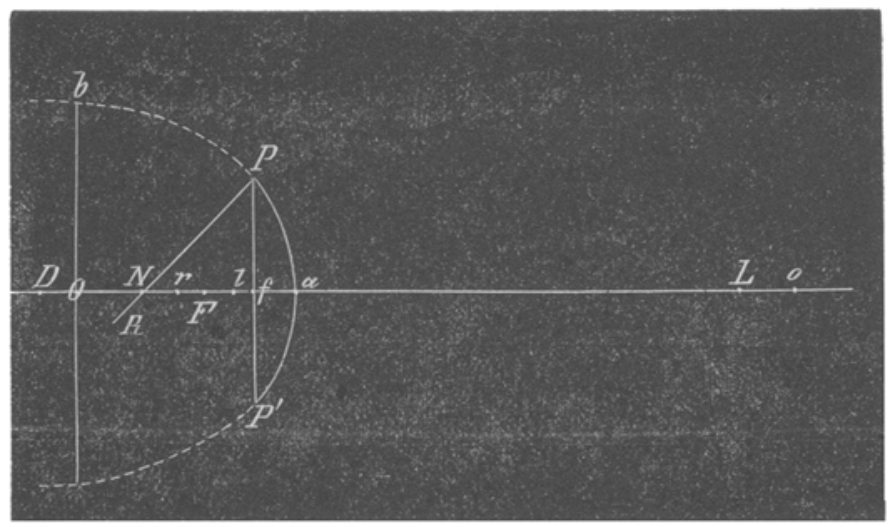

$0 \mathrm{a}=$ halbe grosse Axe $11,5 \mathrm{Mm}$.

$\mathrm{Ob}=$ halbe kleine Axe $9,5 \mathrm{Mm}$.

F der Brennpunkt der Ellipse.

P a $\mathrm{P}^{\prime}$ stellt das der Cornea entsprechende Stiick der Ellipse dar.

P P' ist der Durchmesser der Basis der Cornea $=11,8 \mathrm{Mm}$.

PNR ist die im Punkt $\mathrm{P}$ errichtete Normale.

$\mathrm{N}$ der Durchschnittspunkt der Normalen mit der $\mathrm{X}$-Axe.

$\mathrm{PN}=$ Länge der Normalen.

$\mathrm{PR}=$ Krümmnngsradius im Punkt $\mathrm{P}$.

$a=$ Scheitelpunkt der Cornea.

$\mathrm{a} \mathbf{r}=$ Krümmungsradius im Scheitelpunkt $a$.

In L, weit vor dem Auge, befindet sich das Leuchtobject, direct hinter ihm in 0 das Auge des Beobachters. Das Reflexbild liegt in 1 um den halben Krümmungsradius a $r$ hinter $a$. $y, a$ und $b$ sind bekannt, daher findet $\operatorname{man} \mathrm{Of}=\mathrm{x}=9,01$. Damit kennt man auch a $\mathrm{f}_{\text {, }}$ den Abstand des Hornhautscheitels von der Basis corneae, 
denn af $=0 \mathrm{a}-\mathrm{Of}=11,5-9,01=2,49$. Die Entfernung des Brennpunktes $F$ von $0=\mathrm{c}$ beträgt 6,48 , wie sich dieses ans der Gleichung $a^{2}-b^{2}=c^{2}$ ergiebt.

Der Krümmungsradius im Scheitel der Ellipse berechnet sich aus der Gleichung $r=\frac{b^{2}}{a}$ zu $7,84 \mathrm{Mm}$. Das Spiegelbild 1 liegt daher 3,92 Mm. hinter a.

Was schliesslich die Normale in Punkt P anlangt, so ist die allgemeine Gleichung der Normalen eines Punktes $P$ der Ellipse mit den Coordinaten $x^{\prime} y^{\prime}$ :

$$
y-y^{\prime}=\frac{a^{2} y^{\prime}}{b^{2} x^{\prime}}\left(x-x^{\prime}\right)
$$

Setzt man für $x^{\prime}$ und $y^{\prime}$ die Coordinaten des Punktes $\mathrm{P}$ am Cornealrande ein, so hat man damit die Gleichung der Normalen in P. - Für den Durchschnittspunkt dieser Normalen mit der $X$-Axe $N$ wird $y=0$ und $\mathrm{x}=2,86^{*}$ ).

Was schliesslich die Grösse des zu Punkt $\mathrm{P}$ zugehörigen Krümmungsradius betrifft, so berechnet sich dieser aus der Gleichung

$$
\rho^{2}=\left(\frac{a^{4} y^{2}+b^{4} x^{2}}{a^{8} b^{8}}\right)^{3}
$$

$\mathrm{PR}=\varrho$ ist hiernach, wenn für $\mathrm{x}$ und $\mathrm{y}$ die Coordinaten des Punktes $\mathrm{P}$ eingesetzt worden $=10,047 \mathrm{Mm}$.

Der Drehpunkt möge $13,0 \mathrm{Mm}$. hinter dem Scheitel der Hornhaut liegen.

Wie oben erwähnt, wird sich der Einfluss der Ellipticität der Cornea auf die Lage des Reflexbildes dann am meisten geltend machen, wenn der am weitesten von

*) Nach der Gleichung für den Durchschnittspunkt der Normalen mit der $\mathrm{X}$-Axe $\mathrm{X}=\frac{\mathrm{c}^{2}}{\mathrm{a}^{2}} \mathrm{x}^{\prime} ;\left[\frac{\mathrm{c}^{2}}{\mathrm{a}^{2}}=\varepsilon^{2} ; \varepsilon\right.$ die numerische Excentricität]. 
der Hornhautmitte entfernte Punkt, also ein Punkt am Rande der Cornea spiegelt.

Man kann nun fragen, wann wird im gegebenen Falle der Punkt P am Rande spiegeln - eine wie grosse Drehung muss das Auge ausführen, damit Strahlen, welche von $L$ ausgehen und den Punkt $P$ treffen, an diesem eine solche Reflexion erleiden, dass sie nach L, resp. in das dicht bei $L$ befindliche Auge o zurückgeworfen werden? und wo liegt in diesem Fall das Reflexbild?

Der Punkt $P$ wird dann spiegeln, wenn, wie aus umstehender Figur ersichtlich ist, die ihm zugehörige Normale durch $L$ geht - und das Reflexbild wird dann um den halben dem Punkt $\mathrm{P}$ zugehörigen Krümmungsradius oder 5,023 Mm. hinter $\mathrm{P}$ liegen.

Um die hier in Betracht kommenden Verhältnisse leicht übersehen zu können, beschreibe man aus dem Drehpunkt $D$ einen Kreisbogen mit einem Radius von $1513 \mathrm{Mm}$. durch L (die Entfernung a L möge $=1500 \mathrm{Mm}$. sein), so wird die Normale in Punkt $\mathrm{P}$ den so gezogenen Kreisbogen in S schneiden.

Soll die Normale in P RNPS durch $L$ gehen, so muss das Auge eine Drehung um den Winkel $(\varphi+\beta)$ ausführen. Bei dieser Bewegung beschreibt der Punkt P ein Stück eines Kreisbogens mit dem Radius. D P. Nach ausgeführter Bewegung wird or sich in $P_{2}$ befinden.

Die Grösse des Winkels $\varphi$ ist leicht zu ermitteln:

$$
\begin{aligned}
& \mathrm{Pf} \\
& \overline{\mathrm{Df}}=\operatorname{tg} \varphi \\
& \mathrm{Pf}=\text { halbe Breite der Basis corneae }=5,9 \\
& \mathrm{Df}=\mathrm{D} 0+0 \mathrm{f}=1,5+9,01 \\
& \Varangle \varphi=29^{\circ} 18^{\circ} 30^{\prime \prime} .
\end{aligned}
$$


Nachdem man jetzt den Winkel $\varphi$ kennt, kann man im Dreieck D P f die Seite D P bestimmen, indem

$$
\begin{aligned}
& \frac{\mathrm{Pf}}{\mathrm{DP}}=\sin \varphi \text { ist } \\
& \mathrm{DP}=12,053 .
\end{aligned}
$$

Fig. 3.

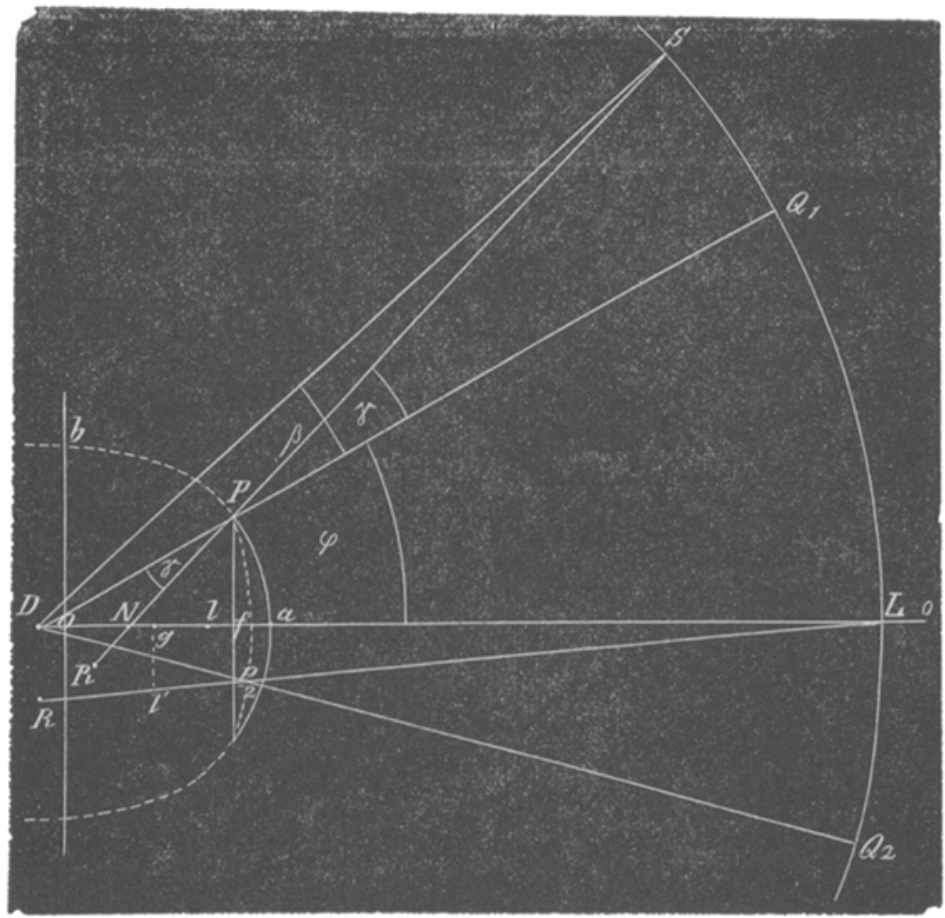

Ưm $\beta$ zu fiuden, bestimnt man zunähst in Dreieck DP $N$ den Winkel $\gamma$. In diesem Dreieck sind die Seiten $\mathrm{D} P$ und $\mathrm{D} \mathrm{N}$ bekannt. $\mathrm{DN}$ ist $=\mathrm{D} \mathrm{O}+\mathrm{ON}=1,5+2,86$. Die 3. Seite N P, die sogenannte Länge der Normalen findet man nach der Gleichung: 


$$
N^{2}=\frac{145}{a^{2}}\left(a^{2}-c^{2} x_{r}^{2}\right)
$$

worin $\varepsilon$ die numerische Excentricität bedeutet $\left(=\frac{c}{a}\right)$.

Für $x_{1}$ die Abscisse des Punktes $P$ eingesetzt, erhält man für $\mathrm{NP}=\mathrm{N}$ den Werth 8,524*). Im Dreieck D P N sind somit die 3 Seiten bekannt und der Winkel PD N $=\Varangle \varphi$. Nach dem Sinussatz findet man daraus $\Varangle \gamma=14^{0} 31^{\prime} 2^{\prime \prime}$. Nun ist Winkel $\beta=\gamma-\Varangle$ D S P. Letzterer Winkel ist mit Hilfe des Sinussatzes aus Dreieck D PS zu finden

$$
\begin{aligned}
& \frac{\sin \mathrm{DSP}}{\sin \mathrm{DPS}}=\frac{\mathrm{DP}}{\mathrm{DS}} \\
& \mathrm{DP} \text { ist }=12,053, \mathrm{D} \mathrm{S}=1513 \text { und } \\
& \Varangle \mathrm{DPS}=180^{\circ}-\gamma \\
& \Varangle \mathrm{D} \mathrm{SP}=0^{\circ} 6^{\prime} 52^{\prime \prime} \\
& \Varangle \beta \text { also }=\Varangle \gamma-\Varangle \mathrm{D} \mathrm{SP}=14^{\circ} 24^{\prime} 10^{\prime \prime} .
\end{aligned}
$$

Damit der auf dem Rande der Cornea liegende Punkt $P$ zur Spiegelung komme, muss demnach das Auge eine Drehung um $43^{\circ} 42^{\prime} 40^{\prime \prime}$ ausführen. - Das Reflexbild wird jetzt in $\mathrm{I}^{\prime}$ liegen, um den halben dem Punkt $\mathrm{P}$ zugehörigen Krümmungsradius hinter $\mathrm{P}$, resp. da $\mathrm{P}$ jetzt nach ausgeführter Bewegung in $P_{2}$ liegt, hinter $P_{2}$.

Für das in o befindliche Auge des Beobachters erscheint das Reflexbild um die Grösse $\mathrm{gl}$, verschoben. Wie gross ist $\mathrm{gl}^{\prime}$ ?

Im Dreieck $\mathrm{gl}^{\prime} \mathrm{L}$ ist $\frac{\mathrm{gl}^{\prime}}{\mathrm{l}^{\prime} \mathrm{L}}=\sin \mathrm{DL} \mathrm{l}^{\prime}$

$l^{\prime} \mathrm{L}=\mathrm{l}^{\prime} \mathrm{P}_{2}+\mathrm{P}_{2} \mathrm{~L}=5,0235+1501,3=1506,3235$ da $I^{\prime} P_{2}=\frac{\varrho}{2}$ und $P_{2} L=P S$ ist; PS findet man aber

*) Oder man sucht den Werth N P mit Hilfe des Cosinussatzes. 
nach dem Sinussatz aus Dreieck D P S

$$
\Varangle \mathrm{D} \mathrm{Ll} l^{\prime}=\Varangle \mathrm{PSD}=0^{\circ} 6^{\prime} 52^{\prime \prime} \text {. }
$$

Es ist daher

$$
\begin{aligned}
& \mathrm{g} \mathrm{l} \mathrm{l}^{\prime}=\sin 6^{\prime} 52^{\prime \prime} \cdot 1506,3235 \\
& \mathrm{~g} \mathrm{l} l^{\prime}=3,008 .
\end{aligned}
$$

Hätte man diese Grösse der Verschiebung gemessen und aus ihr bei bekanntem Drehungswinkel nach der obigen Formel den Abstand des Drehpunktes vom Krümmungsmittelpunkt $\mathrm{zu}$ bestimmen versucht, so hätte man nach der obigen Formel:

$$
\begin{aligned}
& \frac{3,0}{x}=\sin 43042^{\prime} 40^{\prime \prime} \\
& x=4,34
\end{aligned}
$$

gefunden, während doch der Annahme nach der Drehpunkt 5,2 $\mathrm{Mm}$. hinter dem Krümmungsmittelpunkt des Scheitels liegt. In Fälen, die, wie Donders angiebt, van Rees berechnete, konnte der Fehler 2, selbst $3,6 \mathrm{Mm}$. betragen; es mussten dies Beispiele gewesen sein, in denen die Excentricität sehr gross genommen war.

Nachdem Donders diese Fehlerquelle erkannt hatte, verliess er diese Methode. Spätere Drehpunktsbestimmungen führte er in Gemeinschaft mit Doijer in der bekannten Weise aus, dass - nachdem zuvor der Durchmesser der Basis corneat bestimmt war -- bestimmt wurde, um einen wie grossen Winkel das Auge zur Seite gedreht werden musste, damit der eine oder andere Cornealrand gerade hinter ein vertikal gespanntes Haar zu stehen kam.

Aus Drehungswinkel und bekannter Breite der Basis corneae berechnete man nach einfacher Relation*) den

*) Donders u. Doijer, Arch. f. Holl. Beiträge, Bd. III. S. 267. (Die halbe Breite der Basis Corneae zu gesnchtem Abstand des Drehpunktes von der Hornhautbasis ist gleich der Tang. des halben Drehungswinkels. 
Abstand des Drehpunktes von der Basis corneae. Indem man dann in allen Fällen 2,6 Mm. als Entfernung des Hornhautscheitels von Basis hinzuzählte*), erhielt man den Abstand des Drehpunktes von dem Scheitel der Hornhaut. Nach den von Helmholtz**) an 3 Augen ausgeführten Messungen beträgt dieser Abstand im Mittel 2,53 $\mathrm{Mm}$. Nach Messungen, die Jäg e ${ }^{* * *}$ ) an 12 Cadaveraugen ausführte, ergiebt sich im Mittel 2,358. Dabei sind die Differenzen innerhalb der Reihe sehr bedeutend $\dagger$ ). Kn app giebt als Mittel 2,77 an. Daraus ist jedenfalls das eine ersichtlich, dass diese Grösse ziemlich grossen Schwankungen unterliegt. Und häufig dürte, indem in allen Fâllen $2,6 \mathrm{Mm}$. hinzugezählt wurde, zu viel zugezählt worden sein.

Viel wichtiger als diese kleine Ungenauigkeit ist ein anderer Umstand, der bei der Donders-Doijer'schen Methode in Betracht kommt. Die Bewegungen, die das Auge ausführen muss, sind sehr grosse. Mit stark seitlicher Blickrichtung treten nun einmal leichter Kopfbewegungen ein. Um den Einfluss dieser auszuschliessen, lässt Donders freilich den Untersuchten einigemal rasch hinter einander das rechts und links stehende Visirzeichen fixiren, bei deren Fixation dann abwechselnd der eine oder andere Cornealrand hinter dem Haar stehen muss. Dann könnte auch das, worauf J. J. Müller und Berlint广) aufmerksam gemacht haben, bei stark seitlicher Blickrichtung in Betracht kommen. Nach den Untersuchungen dieser findet bei starker Lidöffnung ein Hervortreten des Bulbus statt, wodurch der Drehpunkt

*) Donders, Anomalien der Refract. u. Accommod. S. 159.

**) Arch. f. Ophth. Bd. I, Abth. 2.

***) Ueber die Einstellungen des dioptr. Apparates, S. 276.

†) Maximum $=2,6$; Minimum $=2,1 \mathrm{Mm}$.

†) J. J. Müller, Arch. 士. Ophth. Bd. XIV, 3, s. 183-218. Berlin, Arch. f. Ophth. 1871. S. 159. 
seine absolute Lage im Raum ändert. „Wenn nun bei stark seitlicher Blickrichtung die Pupille sich dem Lidwinkel nähert, und hier theilweise durch die Cilien gedeckt ist, so tritt zum Zweck deutlicheren Sehens ein stärkeres Oeffnen der Lidspalte ein und damit ändert sich durch Hervortreten des Bulbus die absolute Lage des Drehpunktes."

Das Wichtigste aber ist, dass, wie auch Donders sagt, ,in manchen Fällen, besonders bei Myopen*), die Beweglichkeit zu beschränkt ist, um die Cornea den erforderlichen Raum durchlaufen zu lassen."

In solchen Fällen half sich Donders in der Art, dass er statt ei nes Fadens 2 Parallelfäden spannte. Das Auge musste dann so lange zur Seite gewendet werden, bis einwal der eine Cornealrand hinter den einen, das andere Mal der andere Cornealrand hinter den anderen Faden zu stehen kam. Dabei wurden die Drehungen des Auges bestimmt, die nöthig waren, um den einen oder anderen Cornealrand hinter den betreffenden Faden zu bringen. Die Entfernung der beiden Fäden war genau gemessen. „Um den durchlaufenen Raum zu kennen, war es nur nöthig, den Abstand der Fäden von der zuvor gefundenen Breite der Hornhaut abzuziehen und dieser Werth wurde nun ferner der Berechnung zu Grunde gelegt"**).

Die einfache Betrachtung, welche der DoudersDoijer'schen Methode za Grunde liegt, gilt aber, genau genommen, nicht mehr, wenn die Bestimnungsart dadurch modificirt wird, dass 2 Parallelfäden statt eines verwendet werden.

Bevor ich näher auf diesen Punkt eingehe, erinnere ich an einen bekannten Satz aus der Trigonometrie.

*) Unter 10 hochgradigen Myopen war $7 \mathrm{Mal}$ die Beweglichkeit beschränkt. Donders u. Doijer 1. c., S. 271.

**) Donders und Doijer 1. c., S. 267. 
Wenn ein Winkel von $0^{\circ}$ bis $90^{\circ}$ allmälig wächst, so wächst der Sinus anfangs sehr rasch, aber dann immer langsamer.

Stellt nun a einen Punkt an Rande der Cornea dar, D den Drehpunkt und $h$ den Querschnitt des Fadens, welcher gerade in der verlängerten Hornhautaxe liegt, so erscheint für ein weit vor dem Auge gelegenes Auge der Abstand des Cornealrandes von dem Faden $=\alpha \mathrm{h}=\mathrm{af}$.

Fig. 4.

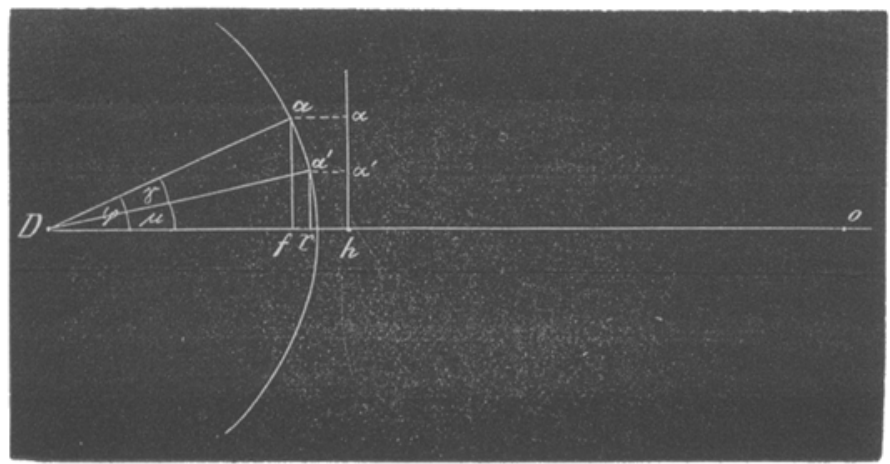

Wenn das Auge jetzt gedreht wird, so wird der Punkt a sich auf einem Kreisbogen, beschrieben mit Radius a $D$, bewegen.

Befindet sich der Punkt $a$ in $a^{\prime}$, so erscheint er für 0 um die Grösse $\alpha^{i} \mathrm{~h}=\mathrm{a}^{t} \mathrm{r}$ von $\mathrm{h}$ entfernt.

Ist nun $\alpha^{t} \mathrm{~h}$ gerade halb so gross wie $\alpha \mathrm{h}$, so geht aus dem erwähnten Satz hervor, dass Winirel $\gamma>$ Winkel $\mu$ sein muss.

$$
\frac{\mathrm{a}^{\prime} \mathrm{r}}{\mathrm{a}^{\prime} \mathrm{D}}=\sin \mu \text {. }
$$

Damit der Sinus doppelt so gross werde, genügt es nicht, den Winkel aufs doppelte wachsen zu lassen, sondern dieser muss um mehr wachsen. 


$$
\begin{aligned}
& \Varangle \varphi>2 \mu \\
\text { oder: } & \gamma>\mu .
\end{aligned}
$$

Nachdem dies vorausgeschickt worden ist, möge der Fall betrachtet werden, in welchem wegen beschrănkter Beweglichkeit 2 Parallelfäden $h$, und $h_{2}$ gespannt werden müssen. Der Abstand der Fäden betrage $4 \mathrm{Mm}$., die gewählten Grössenverhältnisse des Auges und die Bezeichnungen seien dieselben wie oben.

D P der Abstand des Drehpunktes von dem Punkt $P$ am Rande der Cornea ist also $=12,053$, der Durchmesser der Hornhaut $=11,8, \mathrm{Pf}$ also $=5,9$. Der Winkel P D $=\Varangle \varphi$ beträgt nach dem obigen $29^{\circ} 18^{\prime} 30^{\prime \prime}$. Die Hornhautaxe balbirt den Abstand der Fäden $h_{1} h_{2}$.

\section{Fig. 5 .}

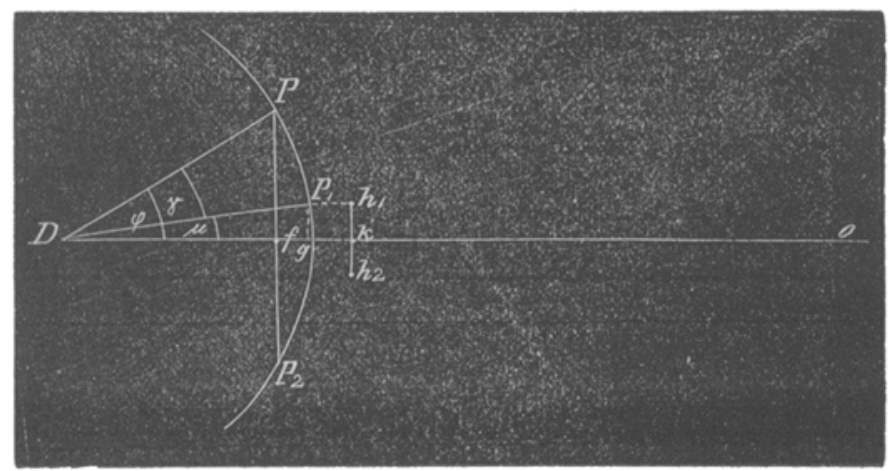

Damit nun Punkt $\mathrm{P}$ hinter den Faden b. zu stehen kommt, muss $\mathrm{P}$ nach $\mathrm{P}^{i}$ sich bewegen. Wie gross ist nun Winkel P D P $=\Varangle \gamma$ ?

$$
\Varangle \varphi=\Varangle \gamma+\Varangle \mu,
$$

Winkel $\mu 1^{\text {ässt }}$ sich aber leicht bestimmen

$$
\begin{aligned}
& \frac{\mathrm{P}^{\prime} \mathrm{g}}{\mathrm{D} \mathrm{P}^{\prime}}=\sin \mu \\
& \mathrm{P}^{\prime} \mathrm{g}=\mathrm{h}^{\prime} \mathrm{k}=2 \\
& \mathrm{D} \mathrm{P}^{\prime}=\mathrm{D} \mathrm{P}=12,053 \\
& \Varangle \mu=9^{0} 33^{\prime} 5^{\prime \prime} .
\end{aligned}
$$


Daher ist Winkel $\gamma=19^{\circ} 45^{\prime} 25^{\prime \prime}$. Eine gleich grosse Drehung muss das Auge im gegebenen Fall $\left(h_{1} h_{2}\right.$ wird durch die Hornbautaxe halbirt) in entgegengesetzter Richtung ausfuhren, damit ein Punkt $P_{2}$ auf dem entgegengesetzten Rande der Cornea hinter $h_{2} z u$ stehen kommt.

Hatte man nun den Drehungswinkel $\gamma$ aus der Stellung der Visirzeichen gefunden und den Raum, den der Punkt durchlaufen hatte, dadurch bestimmt, dass man von der Breite der Hornhaut den Abstand der Fäden abzog und die Differenz der Rechnung zu Grunde legte, um daraus den gesuchten Abstand des Drehpunktes von der Basis corneae za finden, so würde man erhalten

$$
\frac{P f-P^{\prime} g}{x}=\frac{5,9-2}{x}=\operatorname{tg} \gamma
$$

worin $x$ den gesuchten Abstand $D f$ bedeutet - oder:

$$
\begin{aligned}
& x=3,9 \cdot \operatorname{cotg} 19^{\circ} 45^{\prime} 25^{\prime \prime} \\
& x=10,866 .
\end{aligned}
$$

Man findet hiernach also für den gesuchten Abstand 10,866; während derselbe doch in Wirklichkeit (der Annahme nach) 10,51 beträgt, einen Werth, welcher um 0,36 Mm. zu gross ist*).

Kommt zu diesem Fehler noch der kleine oben erwähnte Fehler, der dadurch entstehen kann, dass in allen Fällen 2,6 als Abstand des Hornhautscheitels von Hornhautbasis hinzugezählt wird, welcher Werth aber keineswegs so constant zu sein scheint und wohl häufig kleiner ist als 2,6 Mm., so dürte damit ein Fehler eingeführt werden, der zu gross ist, als dass er nicht beachtet werden darf.

*) Bei Donders und Doijer's Messungen betrug gewöhnlich der Abstand der Fäden 3,02 Mm. Würde dieser Abstand statt des gewählten 4 gesetzt, so würde man im gegebenen Fall für $\times 10,82$ finden. Winkel P D P wäre hier $=22^{\circ} 6^{\prime} 40^{\prime \prime}$. 
Daraus ist ersichtlich, dass das einfache der Donders Doijer'schen Methode zu Grunde liegende Princip und die auf diesem basirende Bestimmung des Drehpunktes nicht mehr unbedingte Anwendung finden, wenn statt eines Fadens 2 Parallelfäden vor das beobachtete Auge gebracht werden, wie dieses bei Fällen von beschränktër Beweglichkeit nöthig, und nun der eine Cornealrand hinter den einen, der andere hinter den andern gestellt wird. - Der hierbei entstehende Fehler beträgt im berechneten Fall 0,36 $\mathrm{Mm}$.

Mit anderweitigen Messungen beschäftigt, war es mir wünschenswerth, im untersuchten Auge auch jedesmal die Lage des Drehpunktes zu kennen. Da diese Messungen sich vorzugsweise auf myopische Augen beziehen, so wünschte ich eine Methode zu besitzen, die bei Genauigkeit und leichter Ausführkeit keine grossen Drehbewegungen erfordert.

Eine solche Methode glaube ich darin zu haben, dass ich die Verschiebung des Cornealreflexes bei Bewegung des Auges messe (wie dies auch schon von $J$ unge und Donders geschehen ist), dabei aber eine derartige Anordnung treffe, dass die Ellipticität der Cornea keinen störenden Einfluss geltend machen kann. Ich gehe aus von der Stellung des Auges, in der die Hornhautaxe in die Richtung der Ophthalmometeraxe fält. Die Gesichtslinie wird dann einen mehr oder weniger weit seitlich von dem Fernrohr gelegenen Punkt treffen. Das Bild eines dicht über dem Fernrohr des Ophthalmometers befindlichen Lichtes wird in der Mitte der Hornhaut liegen.

Wirả jetzt das Visirzeichen seitlich verschoben und führt das untersuchte Auge, indem es fortfährt, dasselbe zu fixiren, eine seitliche Bewegung aus, so wird im Sinn der Drehung auch das Licht verschoben und zwar so, 
dass dasselbe immer in der Verlängerung der Hornhautaxe bleibt.

Dies lässt sich num, wie sich aus Folgendem ergiebt, leicht bewerkstelligen.

Die Messung geschieht folgendermassen:

An der schmalen Seite eines grossen Tisches befindet sich eine Vorrichtung, welche ähnlich der am Förster'schen Perimeter zur Stütze des Kinns dient. Ein kleiner vertikaler Stab komint dabei mit seinem Ende wider den unteren Orbitalrand zu stehen.

Auf dem Tisch, ungefähr unter dem Drehpunkt des untersuchten Auges, befindet sich ein vertikaler Stift, um welchen eine 1 Meter lange Alhydade beweglich ist, die mit ihrem vorderen Ende einen auf den Tisch gezeichneten Gradbogen beschreibt. An dem vorderen Ende dieser Alhydade befindet sich ein Querstück, das an seiner nach dem Gradbogen sehenden Seite gleiche Krümmung hat wie dieser. Ein dem unteren genau in Form und Grösse entsprechendes oberes Querstück ist mit dem unteren durch 2 kleine Säulen verbunden, deren Höhe so gewählt ist, dass das obere Querstück etwa in die Höhe des untersuchten Anges kommt. An beiden Querstücken ist an der gekrümmten Seite eine Gradeintheilung so angebracht, dass die obere der unteren entspricht.

An der Stelle des Nullpunktes ist an dem oberen Querstück ein Licht anzubringen möglich. Längs der gekrümmten Kante lässt sich ferner ein Korkreiter hin. und hersehieben, welcher an einer vertikalen Nadel ein verschiebliches Kügelchen trägt, das als Fixationszeichen dient. Das Ganze stellt somit einen kleinen Tisch dar, welcher dem Ende der Alhydade aufsitzend, auf einer Kreisperipherie hin- und hergeschoben werden kann, welche mit der Länge der Alhydade als Radius beschrieben ist. 
Dicht hinter demselben (unter dem oberen Querstück) befindet sich das Fernrohr des Ophthalmometers; das Licht sitzt diesem somit nicht direct auf, sondern ist durch die Dicke des Querstäckes von dem Fernrohr getrennt, was weiter keinen erheblichen Nachtheil bringt, wenn man das Licht nur recht klein wählt.

Zunächst wird der Nullpunkt des Querstücks auf den Nullpunkt des auf dem Tisch liegenden Gradbogens gebracht (das Fernrohr des Ophthalmometers steht genau unter dem Nullpunkt des oberen Quertheils), und das Fixationszeichen so lange verschoben, bis das Cornealbild des Lichtes genau in der Mitte der Hornhaut liegt*). Aus dem Abstand des Fixationszeichens von dem Nullpunkt erhält man direkt, in Graden ausgedrückt, annähernd den Winkel zwischen Hornhautaxe und Gesichtslinie und aus der Grösse der Drehung der Platte des Ophthalmometers, welche nöthig ist, um die verdoppelten Reflexbilder gleichzeitig auf entgegengesetzte Hornhautränder einzustellen**), findet man weiter die halbe Breite der Cornea.

Nimmt das Auge die gewünschte Stellung ein, so wird, nachdem der Kopf des Untersuchten möglichst gut fixirt ist, - ein vertikal gespanntes Haar, das mit seinem Träger durch eine Mikrometerschraube seitlich verschoben werden kann, - gerade vor das (in der Mitte der Cornea gelegene) Reflexbild des Lichtes gebracht.

Ist dies geschehen, so wird das Tischehen längs des Gradbogens um eine bestimmte Anzahl Grade (gewöhnlich $8^{\circ}-10^{\circ}$ ) verschoben und der Untersuchte dabei angewiesen, fortwährend das Visirzeichen zu fixiren. Licht und Visirzeichen bleiben dabei in derselben Lagebeziehung

*) Siehe oben.

**) Criterium dafür, dass das Reflexbild in der Mitte der Hornhaut liegt. S. oben. 
zu einander und so bleibt das Licht immer in der verlängerten Hornhautaxe.

Nach ausgeführter Bewegung erscheint das Reflexbild seitlich vom Haar. Die Entfernung von Haar zu Reflexbild ist die Grösse der Verschiebung. Sie wird gemessen, indem man die Platten des Ophthalmometers so lange dreht, bis das erste verdoppelte Reflexbild mit dem zweiten Haar zusammenfällt.

Um zu sehen, ob keine Kopfbewegung stattgefunden hatte, wurde das Tischchen wieder auf den Nullpunkt gebracht. War die Drehung der Platten geblieben, -so musste dann, wenn keine Bewegung stattgefunden hatte, hinter je einem Haar ein Reflexbild stehen. Zur Controle wurde dann das Tischchen um eine gleiche Anzahl Grade nach der entgegengesetzten Seite verschoben. Bei unverändert gebliebener Stellung der Ophthalmometerplatten musste jetzt hinter einem Haar und zwar dem anderen als bei der ersten Drehung um 10\%, ein Reflexbild liegen, und bei abermaligem Einstellen auf den Nullpunkt musste wieder hinter jedem Haar ein Reflexbild stehen, wenn mittlerweile keine Kopfbewegung stattgefunden hatte. Aus einer Anzahl solcher sehr genauen Bestimmungen wurde dann das Mittel genommen.

Wie schon oben erwähnt wurde, verfuhr Donders bei der Messung der Verschiebung des Reflexes derart, dass er nicht jedesmal das Haar vor das Reflexbild in der Primärlage brachte, sondern, wenn dieses vor der Drehung etwas zur Seite des Haares lag, er nach ausgeführter Drehung die Grösse der dabei stattgefundenen Verschiebung mass, indem er die Platten des Ophthalmometers so lange drehte, bis das erste Reflexbild um eine gleiche Grösse vom zweiten Haare abwich. Bei rasch abwechselnder Fixation der Visirzeichen lasse sich dies mit der nöthigen Genauigkeit erreichen. - Letztere Messungsart hat jedenfalls den Yorzug der leichteren 
Ausführung. Da bei ihr aber die Genauigkeit der Messung von der richtigen Schätzung des Abstandes des Reflexbildes von dem Haar abhängt und es zur Erlangung der nöthigen Fertigkeit Uebung bedarf, so schien es mir bei den ersten Messungen zweckentsprechender; um vollständig zuverlässige Werthe zu erhalten, das bezeichnete, freilich oft für den Untersuchten lästige Verfahren einzuschlagen, indem es manchmal lange dauerte, bis man Werthe erhielt, die den gestellten Anforderungen vollständig genügten.

Schliesslich musste noch der Krümmungsradius im Scheitel gemessen werden, was auf die bekannte Art mit dem Ophthalmometer geschah. War dies geschehen, dann hatte man alle Werthe, um die Lage des Drehpunktes berechnen zu können.

Nachdem ich das Verfahren der Messung beschrieben habe, bleibt mir die Aufgabe, zu zeigen, welche Vortheile dasselbe hat und insbesondere, dass bei dor beschriebenen Anordnung die Ellipticität der Cornea keinen, resp. leinen nennenswerthen Einfluss auf die Verschiebung des Reflexbildes hat.

$\mathrm{Zu}$ diesem Zweck möge der Fall betrachtet werden, in welchem das Ange eine Drehung von $20^{\circ}$ ausführt, wobei das Licht, im Sinn der Drehung des Auges sich mitbewegend, stets in der verlängerten Hornhautaxe bleibt. Der Abstand des Drehpunktes vom Hornhautscheitel betrage $13 \mathrm{Mm}$., die Hornhaut sei der Einfachheit halber zunächst sphärisch angenommen, nach einem Radius von $8 \mathrm{Mm}$. gekrümmt.

Wo liegt jetzt nach ausgeführter Drehung das Spiegelbild der Cornea, wie gross ist seine Verschiebung und welcher Punkt der spiegelnden Oberfläche vermittelt die Spiegelung?

Die Zeichen in Fig. 6 bedeuten dasselbe wie in Fig. 1.

D ist der Drehpunkt, c der Krümmungsmittelpunkt 
der Hornhaut, m deren Scheitel bei der Anfangsstellung, F bedeutet das Fixationszeichen; der Voraussetzung nach geht, wenn die Gesichtslinie durch $\mathrm{F}$ geht, die Hornhautaxe durch $L$. Um die Figur nicht übertüssig 20 compliciren, sind die Gesichtslinien nicht gezeichnet. 1 die Lage des Cornealbildes bei dieser Augenstellung.

Nach ausgeführter Drehung liegt $c$ in $c^{d}, m$ in $\mathrm{m}^{d}$. Blieb das Licht in $\mathrm{L}$, so liegt jetzt das Reflexbild in $\mathrm{l}$;

Fig. 6.

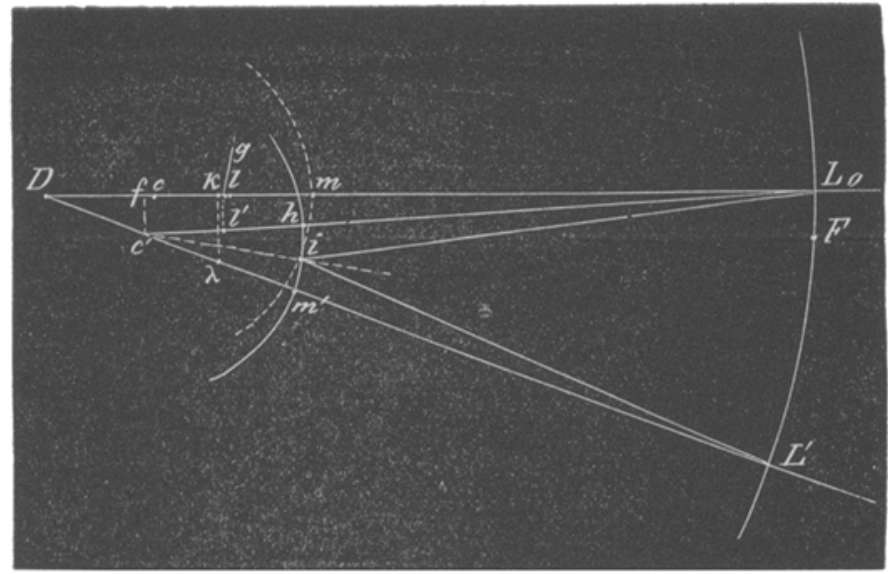

wurde dagegen das Licht im Sinn der Drehung verschoben, so dass es nach der Drehung in $L^{\prime}$ auf der verlängerten Hornhautaxe liegt, so erscheint das Reflexbild in $\lambda$. Betrug im ersten Fall die Verschiebung (für das weit von dem untersuchten Auge befindliche Auge des Beobachters) $\mathrm{gl}^{\mathrm{l}}$, wobei für einen Drehungswinkel von $20^{\circ} \mathrm{g} \mathrm{l}^{\prime}=1,71 \mathrm{Mm}$. ist, so ist im letzteren Fall die Verschiebung gleich $\mathrm{k} \lambda$. Es verhält sich aber $\frac{\mathrm{gl}}{\mathrm{k} \lambda}$ oder, da für $\mathrm{gl}^{\prime}$ nach dem oben Gesagten auch $\mathrm{f} \mathrm{c}^{\prime}$ gesetzt werden kann, 


$$
\frac{\mathrm{fc}^{\prime}}{\mathrm{k} \lambda}=\frac{\mathrm{D} \mathrm{c}}{\mathrm{D} \lambda}=\frac{158}{\mathrm{Dc^{ \prime } + \mathrm { c } ^ { \prime } \lambda}}=\frac{5}{5+4}
$$

Die Verschiebung betrăgt im letzteren Fall also einen nahezu doppelt so grossen Werth - oder mit anderen Worten, um dieselbe Grösse der Verschiebung des Reflexbildes zu erhalten, genügt in letzterem Fall eine Drehung des Auges um wenig mehr als die Hälfte der Anzahl Grade als im ersten Fall.

Die Grösse $k \lambda$ berechnet sich, wie aus der Figur ersichtlich ist aus der Relation

$$
\begin{aligned}
& \frac{\mathrm{k} \lambda}{\mathrm{D} \lambda}=\sin \mathrm{CD} \mathrm{c}^{\prime} \\
& \mathrm{D} \lambda \text { ist }=\mathrm{Dc}^{\prime}+\mathrm{c}^{\prime} \lambda
\end{aligned}
$$

$\mathrm{D} \mathrm{c}^{\prime}$ ist nun der gesuchte Abstand des Drehpunktes vom Krümmungsmittelpunkt der Cornea $=\mathrm{x} ; \mathrm{c}^{\prime} \lambda=$ balber Krümmungsradius.

$$
\frac{\mathrm{k} \lambda}{\mathrm{x}+\frac{\mathrm{r}}{2}}=\sin \mathrm{cD} \mathrm{c}^{\prime} \text { oder } \mathrm{x}=\frac{\mathrm{k} \lambda}{\sin \mathrm{cDc}}-\frac{\mathrm{r}}{\mathrm{z}} \text {. }
$$

Wie oben gezeigt wurde, spiegelte nach ausgeführter Drehung, wenn das Licht an Ort und Stelle geblieben war, ein Punkt $\mathrm{h}$, dessen Lage dadurch bestimmt war, dass er auf der Verbindungslinie von $L$ und $c$ liegt. Der Winkel $\mathrm{h} \mathrm{c}^{\prime} \mathrm{m}^{\prime}$ konnte gleich dem Drehungswinkel gesetzt werden (siehe oben).

Wurde dagegen das Licht mit der Bewegung des Auges nach $L^{\prime}$ geführt, so ist es der Punkt $\mathrm{i}$, welcher Strahlen, die von $L^{\prime}$ ausgehen, in das in o befindliche Auge des Beobachters reflectirt. Die Lage dieses Punktes $i$ ergiebt sich nach den Gesetzen der Spiegelung durch die Halbirungslinie des Winkels $L e^{\prime} L^{\prime}$. Wo diese Linie die spiegelnde Fläche schneidet, da liegt i.

Daraus folgt, dass der Punkt $h$ doppelt so weit vom Scheitel liegt wie der Punkt i.

Der Vortheil der Verschiebung des Lichtes nach $L^{\prime}$ 
ist somit ein doppelter: einmal fällt die Verschiebung des Reflexbildes nahezu doppelt so gross aus und zweitens vermittelt ein Punkt der spiegelnden Oberfläche die Reflexion nach 0 , welcher dem Scheitel der Hornhaut doppelt so nahe liegt.

Hatte man bei einer Drehung um 20, wie erwähnt, bei an Ort und Stelle bleibender Leuchtquelle, eine Verschiebung des Reflexes um 1,71 Mm. erbalten, so erhält man die gleiche Verschiebung schon bei einer Drehung von nur $10^{\circ} 57^{\triangleleft}$ wenn das Licht im Sinn der Drehung gleichmåssig verschoben wurde.

Fig. 7.

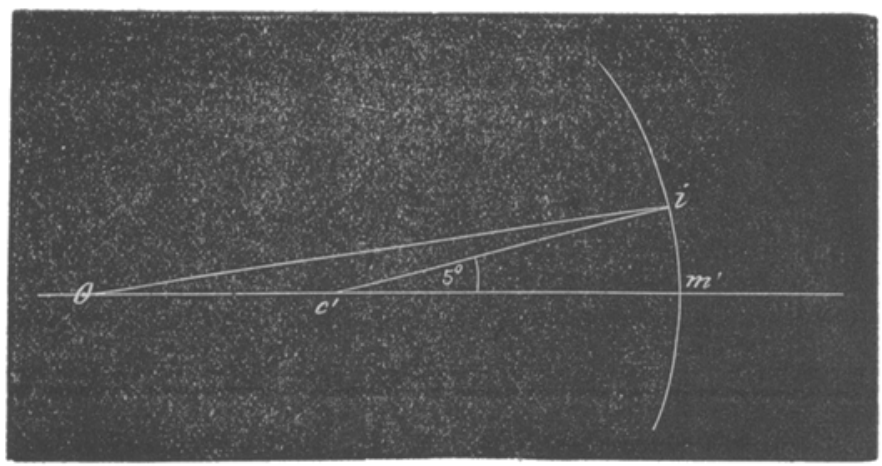

Spiegelte im ersten Falle ein Punkt h, der, wenn seine Entfernung vom Scheitel der Hornhaut in Graden eines Winkels ausgedrückt wurde, dessen Scheitel in $\mathbf{c}^{\prime}$ liegt - um $20^{\circ}$ von $\mathrm{m}^{\prime}$ entfernt lag, so spiegelte im anderen Fall ein Punkt, der nur $5^{\circ} 30^{\prime}$ vom Scheitel liegt.

$\mathrm{Da}$, wie oben gesagt wurde, bei meinen Messungen eine Drehung von nur $8^{\circ}$ resp. $10^{\circ}$ ausgeführt wurde, so spiegelte in diesen Fällen ein Punkt, der $4^{\circ}$ resp. $5^{\circ}$ von $\mathrm{m}^{\prime}$ lag. Drückt man den Abstand des spiegelnden Punktes von dem Hornhautscheitel anstatt in Winkel- 
graden, bezogen auf den Krümmungsmittelpunkt c resp. $\mathbf{c}^{t}$ - in Graden eines Winkels aus, dessen Scheitel in einem Punkt o liegt, welcher sich $11,5 \mathrm{Mm}$. hinter m resp. $\mathrm{m}^{\prime}$ befindet (Mittelpunkt der Ellipse), so erhält man für Winkel $\mathrm{m}^{\prime} \mathrm{O}$ i $3^{0} 28^{\prime} 45^{\prime \prime *}$ ) (Fig. 7).

Die vorstehende Betrachtung bezog sich auf eine sphärisch angenommene Hornhaut. Nun stellt aber ein Horizontalschnitt, welcher durch die Cornea gelegt wird, diese als ein Stück einer Ellipse dar, deren halbe grosse Axe annähernd 11,5 Mm., deren halbe kleine Axe 9,5 $\mathrm{Mm}$. gesetzt werden kann; werden diese Werthe zu Grunde gelegt, wie dies auch oben geschehen ist und fragt man jetzt, welcher Punkt der Ellipse spiegelt, wenn um einen Punkt, der $13 \mathrm{Mm}$. hinter dem Schnittpunkt der Ellipse mit der grossen Axe liegt (d. i. der Drehpunkt des Auges, welcher $13 \mathrm{Mm}$. hinter dem Hornhautscheitel liegend angenommen wird), eine Drehung um $10^{\circ}$ ausgeführt wird und das Licht bei und nach der Bewegung immer auf der verlängerten grossen Axe bleibt?

Es wird dies ein Punkt $P$ sein, der dadurch bestimmt ist, dass die ihm zugehörige Normale den Winkel halbirt, welcher gebildet wird, wenn man von dem nach ausgeführter Bewegung in $L^{\prime}$ befindlichen Licht nach $P$ und von $\mathbf{P}$ nach dem Auge des Beobachters o Linien zieht.

Wie eine einfache Construction zeigt, ist in diesem Fall der Einfallswinkel, das ist der Winkel, welchen $L^{\prime} P$ mit der Normalen in $\mathrm{P}$ bildet, gleich dem Reflexionswinkel, d. i. dem Winkel, welchen o P mit der Normalen bildet. Wird nach dieser Betrachtung, der die Spiegelung vermittelnde Punkt $P$ der Ellipse gesucht, so erhält man einen Punkt, welcher noch etwas näher dem Scheitel

*) Zunächst wird $\mathrm{Oi}$ mittelst des Cos. satzes gesucht, $\mathrm{Oi}=11,49$, und dann mittelst Sinussatz $\Varangle i O \mathrm{~m}$ '. 
gelegen ist. Wird durch denselben ein Strahl aus dem Centrum der Ellipse 0 gezogen, so bildet dieser mit der grossen Axe einen Winkel von circa $3^{\circ}$.

Wie gross ist nun im gegebenen Fall der Krümmungsradius, der diesem Punkte $P$ zugehört? und um wie viel differirt derselbe von dem Krümmungsradius im Scheitelpunkt? Vor Beantwortung dieses speciellen Falles dürfte es zweckmässig sein, im Allgemeinen die Zunahme des Krümmungsradius an verschiedenen Punkten der Ellipse mit deren Abstand vom Scheitel etwas näher zu betrachten.

Fig. 8.

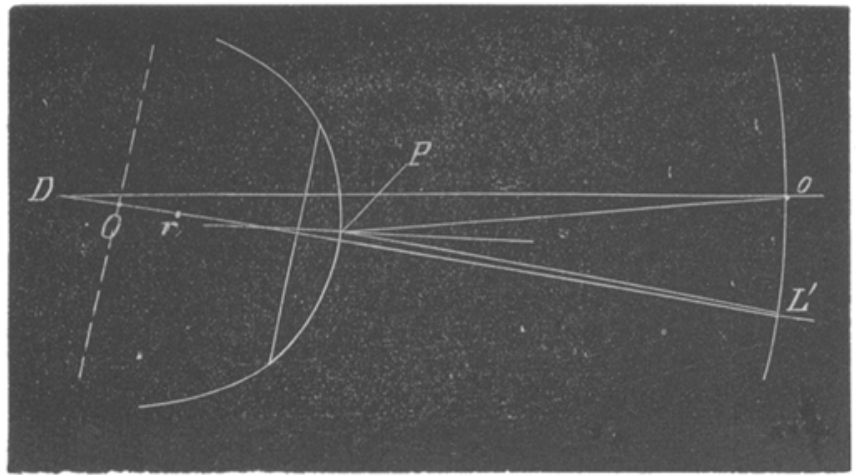

Auch ther sel die melrel wälnte kilpse zu Gruude gelegt, deren halbe grosse Axe 11,5, deren halbe kleine Axe $9,5 \mathrm{Mm}$. beträgt. Der Durchmesser der Hornhaut sei mit $11,8 \mathrm{Mm}$. angenommen.

Wird auch jetzt wieder, wie oben; ein rechtwinkliges Coordinatensystem zu Grunde gelegt und die Ellipse so construirt, dass deren grosse Axe mit der X-Axe zusammenfällt und das Centrum der Ellipse in den Anfangspunkt des Coordinatensystems zu liegen kommt, und werden jetzt aus dem Centrum der Ellipse 0 Strahlen an die Ellipse gezogen, die mit einander je einen Wïnkel von $5^{0}$ einschliessen, so treffen diese die Ellipse in den Punkten $\mathrm{P}, \mathrm{P}^{\prime}, \mathrm{P}^{\prime \prime}, \mathrm{P}^{\prime \prime}$ etc.

พ. Graefe' Arohir fïr Ophthalmologie, XXI. 2. 
Wie gross sind die diesen Punkten zugehörigen Krümmungsradien? Die allgemeine Gleichung für den Krümmungsradius eines Punktes der Ellipse ist:

$$
\varrho^{2}=\frac{\left(a^{4} y^{2}+b^{4} x^{2}\right)^{3}}{a^{8} b^{8}}
$$

Fị.. 9.

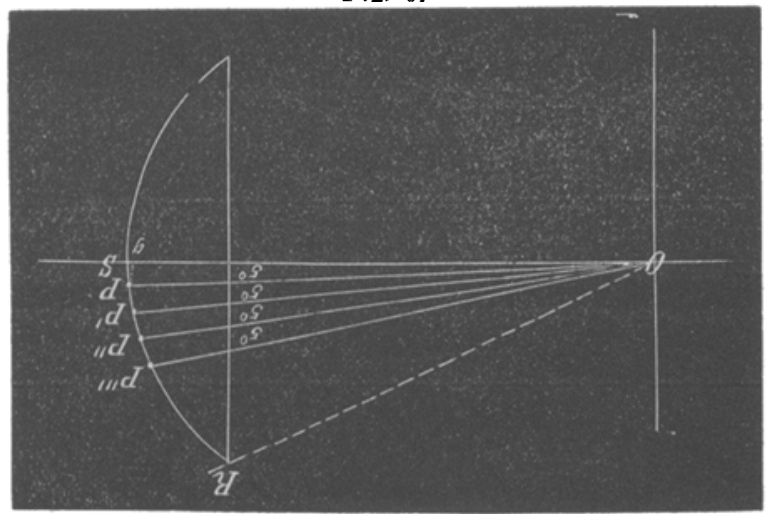

Ist $\mathrm{y}=0$, was für den Puukt $\mathrm{S}$, den Scheitelpunkt, der Fall ist, so vereinfacht sich diese Gleichung in:

$$
\rho=\frac{b^{2}}{a} \text {. }
$$

Die Krümmungsradien der Punkte $\mathrm{P}, \mathrm{P}^{\prime}, \mathrm{P}^{\prime \prime}$ etc. findet man daher, wenn für $x$ und $y$ ihre Coordinaten eingesetzt werden.

Letztere lassen sich aber leicht bestimmen.

Hat man einen beliebigen Punkt $p$ der Ellipse mit den Coordinaten $x y$, so ist $\frac{y}{x}=\operatorname{tg}$ des Winkels, welchen der durch Punkt $p$ aus 0 , dem Centrum der Ellipse, gelegte Strahl mit der grossen Axe bildet. Sei dieser Winkel $\varphi$ genannt, so ist

$$
\left.\frac{\mathrm{y}}{\mathrm{x}}=\operatorname{tg} \varphi^{*}\right)
$$

*) Far Punkt $P$ z. B. $\frac{P g}{0 g}=\operatorname{tg} 5^{\circ}$. 
oder da

$$
\begin{aligned}
& \mathrm{x}=\frac{\mathrm{a}}{\mathrm{b}} \sqrt{\mathrm{b}^{2}-\mathrm{y}^{2}} \text { ist, } \\
& \mathrm{y}^{2}=\frac{\operatorname{tg}^{2} \varphi \cdot \mathrm{a}^{2}}{1+\operatorname{tg}^{2} \varphi \frac{\mathrm{a}^{2}}{\mathrm{~b}^{2}}}
\end{aligned}
$$

Hiernach sind im vorliegenden Fall die Coordinaten des Scheitelpunktes $\mathrm{S}: \mathrm{x}_{\mathrm{B}}=11,5$

$$
y_{8}=0
$$

des Punktes P: . . $\mathrm{x}=11,436$

$$
\mathrm{y}=1,000524
$$

des Punktes $\mathrm{P}^{\prime}$ : . . $\mathrm{x}^{\prime}=11,247$

$$
y^{\prime}=1,983
$$

des Punktes P": . . $\mathrm{x}^{\prime \prime}=10,939$

$$
\mathrm{y}^{\prime \prime}=2,93
$$

und die des Punktes $\mathrm{P}^{\prime \prime}$ :. . $\mathrm{x}^{\prime \prime}=10,523$

$$
\mathrm{y}^{\prime \prime \prime}=3,83 \text {. }
$$

Die Coordinaten eines Punktes $R$ am Rande der Cornea sind nach dem obigen bekannt

$$
\begin{aligned}
& \mathrm{x}_{\mathrm{x}}=9,01 \\
& \mathrm{y}_{\mathrm{r}}=5,9 .
\end{aligned}
$$

Ein Strahl aus $O$ durch $R$ gelegt bildet mit der grossen Axe einen Winkel von $33^{\circ} 13^{\prime} 44^{\prime \prime}$.

Wird jetzt der Krümmungsradius für Punkt $P, P^{\prime}$ u. s. w. berechnet, so findet man:

für den Scheitelpunkt $S=7,84$

für den Punkt $\mathrm{P}$. . $=7,9084$

für den Punkt $\mathrm{P}^{\prime} . .=8,0877$

für den Punkt $\mathrm{P}^{\prime \prime}$. . $=8,3752$

für den Punkt $\mathrm{P}^{\prime \prime \prime} . \quad .=8,748$.

Es. wächst somit der Krümmungsradius

von $\mathrm{S}$ bis $\mathrm{P}$. . um $0,0684 \mathrm{Mm}$.

von $\mathrm{P}$ bis $\mathrm{P}^{\prime}$. . um 0,1793 ",

von $\mathrm{P}^{\prime}$ bis $\mathrm{P}^{\prime \prime}$. um 0,2875 "

von $\mathrm{P}^{\prime \prime}$ bis $\mathrm{P}^{\prime \prime \prime}$. um 0,373 "

Wächst der Winkel bis $33^{\circ} 13^{\prime} 40^{\prime \prime}$, so beträgt der 
Krümmungsradius (für Punkt $\mathrm{R}$ am Rande der Cornea) schon 10,047 Mm.

Aus dieser. Zusammenstellung ist ersichtlich, wie sehr rasch der Krümmungsradius wächst mit weiterer Entfernung vom Scheitel ${ }^{*}$ ) und hieraus geht wiederum der grosse Vortheil hervor, den es hat, wenn ein nahe dem Scheitel der Cornea gelegener Punkt spiegelt.

Kommen wir jetzt auf den erwähnten speziellen Fall zurück; wie gezeigt, spiegelte nach ausgeführter Drehung um $10^{\circ}$ ein Punkt, der dadurch bestimmt ist, dass ein aus dem Centrum der Ellipse durch ihn gelegter Strahl mit der X-Axe einen Winkel von circa $3^{0}$ bildet. Die Coordinaten eines solchen Punktes $\mathrm{x}$ und $\mathrm{y}$ sind 11,477 resp. 0,6014 Mm., der zugehörige Krümmungsradius ist $7,87 \mathrm{Mm}$. gross, differirt gegen den des Scheitelpunktes $(7,84)$ also nur um $\frac{3}{100} \mathrm{Mm}$.

Bei einer so kleinen Differenz kann die Ellipticität der Cornea keinen Einfluss geltend machen, der nicht innerhalb der Fehlergrenze der Messung läge.

Zum Schluss wird der Krüminungsradius im Scheitel der Hornhant bestimmt. So bekannt die Methode der Bestimmung des Krümmungsradius ist, so möchte ich doch an dieser Stelle mit wenigen Worten auf dieselbe zu sprechen kommen.

Senkrecht zur Hornhautaxe wird in einer Entfernung von etwa $8^{\prime}-10^{\prime}$ ein Massstab nahezu in gleicher Höhe mit dem beobachteten Auge aufgestellt. Die Stelle, in welcher die verlängerte Hornhautaxe den Massstab schneidet, ist der Ausgangspunkt, von dem um $2^{\prime}$ nach der einen Seite ein Licht angebracht ist. - Die Mitte

*) Es würde dieses auch ersichtlich sein, wenn man in die Gleichung für den Krümmungsradins Winkel mit ihren Functionen einfuhrte. 
zweier Lichter fällt um ebensoweit vom Ausgangspunkt nach der anderen Seite. Die 3 Lichter stellen das Object dar. Seine Grösse ist die Entfernung von einem Licht bis zur Mitte der beiden anderen. Auf der Cornea erscheint ein Spiegelbild, dessen Grösse mit dem Ophthalmometer gemessen wird.

Den Radius findet man nach der bekannten Formel

$$
\mathrm{r}=\frac{2 \mathrm{a}}{\mathrm{b}} \cdot \beta
$$

worin $\beta$ die Bild- und $\mathrm{b}$ die Objectgrösse bedeutet und a die Entfernung des Objectes von spiegelnder Fläche darstellt. Je grösser a gewählt wurde, desto kleiner ist der kleine Fehler, welcher daraus resultirt, dass die Grösse des Radius gegenüber a vernachlässigt wird.

Angenommen, der Abstand a betrage $2600 \mathrm{Mm}$. bei einer Objectgrösse von $1000 \mathrm{Mm}$., so wird ein Strahl, der von dem Endpunkte des Objects ausgeht, mit der Hornhautaxe einen Winkel von $10^{\circ} 51^{\prime}$ einschliessen.

Fig. 10.

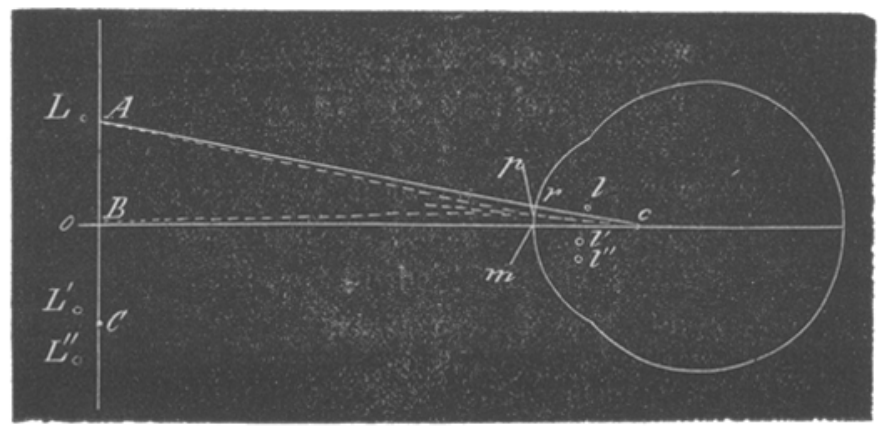

Es ist dieses aus beistehender Figur ersichtlich, $\mathrm{AC}$ ist das $1000 \mathrm{Mm}$. grosse Object, dargestellt durch die 3 Lichter $L, L^{\prime}$ und $L^{\prime \prime}, B \mathrm{~m}$ ist die Entfernung vom 
Auge, B ist der Ausgangspunkt (durch ihn geht die verlängerte Hornhautaxe). Hinter $B$ in $o$ befindet sich das Auge des Beobachters. In c befindet sich das Krümmungscentrum des Scheitels der Cornea. Nun ist

$$
\frac{A B}{B m+m c}=\operatorname{tg} A \circ B \text {, }
$$

oder da $\mathrm{m} c$ gegen $\mathrm{Bm}$ vernachlässigt werden darf,

$$
\begin{aligned}
& \frac{500}{2600}=\operatorname{tg} A \text { c B } \\
& \Varangle A \subset B=10051
\end{aligned}
$$

Das Bild des Lichtes $\mathrm{L}$ wird in 1 liegen. Welcher Punkt der spiegelnden Oberfläche ist es, welcher Strablen die von $L$ ausgehend ihn treffen, so reflectirt, dass sie in das in o befindliche Auge des Beobachters fallen?

Stellte die Hornhaut eine sphärische Fläche dar, so würde der verlängerte Radius in dem spiegelnden Punkt $p$ den Winkel $A p B$ halbiren. $p$ wird in der Mitte von $\mathrm{r}$ und $\mathrm{m}$ liegen. Der Winkel $\mathrm{r} \mathrm{cm}$ beträgt $10^{\circ} 51^{\prime}$, der Winkel $\mathrm{p} \mathrm{c} \mathrm{m}$ daher $5^{\circ} 25^{\prime} 30^{\prime \prime}$.

Da aber die Hornhaut keine sphärische Fläche ist, so wird die Lage des spiegelnden Punktes $p$ dadurch eine andere sein. Der spiegelnde Punkt wird dem Scheitel etwas näher liegen, doch ist diese Differenz sehr klein.

Indem der spiegelnde Punkt etwas von dem Scheitelpunkt der Cornea entfernt liegt, die Cornea aber ellipsoidisch ist, so hat der spiegelnde Punkt, wie dieses mit den Eigenschaften der Ellipse zusammenhängt, einen etwas grösseren Krümmungsradius als der Scheitelpunkt. Die Differenzen sind hier aber so klein, dass man und mit vollem Recht - auch hier kein Bedenken trăgt, die dem Scheitelpunkt nächst benachbarte Partie als nach gleichem Radius gekrümmt zu betrachten.

Bei der obigen Auseinandersetzung war nur immer von Bewegungen in der Horizontalen und der Drehpunktsbestimmung für solche Bewegungen die Rede. Soll 
der Drehpunkt für Vertical-Bewegungen oder bei gesenkter oder erhobener Blickebene bestimmt werden, so braucht man dem Apparat nur die der Blickebene entsprechende Anordnung zu geben. Ich behalte mir vor, auf diese Bestimmungen später zurückzukommen; an dieser Stelle, kam es mir vorzugsweise auf die Darlegung des den Messungen zu Grunde liegenden Princips an, welches seine Anwendung finden soll in allen Fällen von beschränkter Beweglichkeit (also bei der Mehrzahl der höhergradig myopischen Augen), die die Bestimmung mittelst der einfachen Donders-Doijer'schen Methode nicht gestatten. Werden in Fällen beschränkter Beweglichkeit 2 Fäden statt eines gespannt und die Bestimmung demgemäss, wie oben auseinandergesetzt worden, modificirt, so ist die Anwendung des der DondersDoijer'schen Methode (mit einem Faden) zu Grunde liegenden Princips nicht streng richtig, der aus dieser Ungenauigkeit entspringende Fehler ist zu gross, als dass er vernachlässigt werden dürfte. - Der Methode, aus der Verschiebung eines Cornealreflexbildes bei Bewegungen des Auges die Lage des Drehpunktes zu bestimmen, lässt sich eine einfache Anordnung geben, dass die Ellipticität der Cornea auf die Lage resp. die Verschiebung des Reflexes keinen Einfluss geltend machen kann. Indem ich eine Anzahl Drehpunktsbestimmungen anfüge, welche nach dem im Obigen erörterten Principe (bei Bewegungen in der Horizontalen) genommen wurden, erwähne ich, dass die ersten Bestimmungen in dem physiologischen Institut des Herrn Prof. von-Vie rordt gemacht wurden, welcher mir freundlichșt die Räume seines Institutes zur Verfügung stellte, wofür ich ihm an dieser Stelle Dank sage. - $\mathrm{Zu}$ ganz besonderem Danke fühle ich mich auch Herrn Prof. von Reusch gegenüber verpflichtet, welcher mich bei der Construction der Apparate sehr wesentlich unterstützte, welcher Unter. 
stützung ich danke, dass diese die für die Messung nöthige Genauigkeit haben.

Herr stud. N.

I. Bestimmung.

M. $\frac{1}{7,5}$

Pupillendistanz $2^{\prime \prime} 6^{\mu \prime}$.

Bei stark seitlichem Blick Langbau äusserlich erkennbar.

- Sehschărfe $\frac{15}{20}$. - Vordere Kammer tief. - Pupillen weit. - Mit dem Augenspiegel Refraction bestimmt, nahezu gleicher Grad der Myopie gefunden. An beiden Augen nach aussen von der Papille grosse Coni, am rechten Auge etwas weniger gross als am linken.

Winkel zwischen Hornhautaxe und Gesichtslinie nach der Donders'schen Methode bestimmt $=1,78^{\circ}$ (Mittel aus 10 Bestimmungen).

Durchmesser der Basis corneae $=12,0 \mathrm{Mm}$. (Mittel aus 10 Bestimmungen).

Verschiebung des Reflexbildes bei Drehung des Auges um $10^{\circ}=1,74 \mathrm{Mm}$. (Mittel aus 10 Bestimmungen, 5 bei Drehung der Platten nach rechts, 5 bei Drehung der Platten nach links. Grösste Differenz innerhalb der Reihe $\left.=1,9^{\circ}\right)$. Krümmungsradius im Scheitel $=7,74 \mathrm{Mm}$. $\mathrm{x}=$ Abstand des Drehpunktes von dem Krümmungsmittelpunkt des Scheitels:

$$
\begin{aligned}
& \frac{1,74}{x+\frac{r}{2}}=\sin 10^{\circ} \\
& x+\frac{r}{2}=10,02 \mathrm{Mm} .
\end{aligned}
$$

Abstand des Drehpunktes von dem Hornhautscheitel $=\mathrm{x}+\mathrm{r}=13,89 \mathrm{Mm}$.

Bei der Bestimmung am rechten Auge wurde so verfahren, dass bei der Primärstellung das Haar das 
Reflexbild genau deckte. War nach ausgeführter Drehung das Reflexbild seitlich verschoben (zum Haar), so wurden die Platten des Ophthalmometers solange gedreht, bis das erste Hornhautreflexbild mit dem zweiten Haar sich deckte. Bei Rückkehr in die Primärstellung musste dann, wenn keine Kopfbewegung stattgefunden hatte, hinter je einem verdoppelten Haar ein Reflexbild stehen.

Nachdem auf diese Weise am rechten Auge sehr genau der Drehpunkt bestimmt war, hatte ich in dieser genauen Bestimmung eine annähernde Controle für den Grad der Genauigkeit der Messung am linken Auge, an dem die Verschiebung des Reflexbildes nach der Donders'schen Angabe gemessen wurde. Beide Augen hatten gleiche Grade von Myopie und somit war wohl die Annahme berechtigt, dass in beiden Augen der Drehpunkt annähernd gleich weit hinter dem Scheitel lag. Deckte das vertical gespannte Haar das Reflexbild der Cornea bei der Primärstellung nicht, sondern lag letzteres etwas zur Seite, so wurden nach ausgeführter Bewegung die Ophthalmometerplatten so lange gedreht, bis das erste Reflexbild vom zweiten Haar um die gleiche Entfernung abstand, wie das Reflexbild von dem Haar in der Primärstellung. Bewegte sich das Ange einige Mal aus der Primärstellung um $10^{\circ}$ und dann wieder zurück in die Primärstellung (indem das Tischchen vom Nullpunkt um $10^{\circ}$ verschoben und dann wieder auf den Nullpunkt gebracht wurde); so konnte in der That eine etwaige Kopfbewegung sofort erkannt und corrigirt werden.

Es ergaben sich hierbei folgende Werthe:

II. Bestimmung.

Herr stud. N.

Linkes A uge M. $\frac{1}{7,5}$. 
Winkel zwischen Hornhautaxe und Gesichtslinie $=2,2^{\circ}$ (Mittel aus 2 Bestimmungen).

Durchmesser der Basis corneae $=11,9 \mathrm{Mm}$. (Mittel aus 2 Bestimmungen).

Verschiebung des Reflexbildes bei Drehung um $10^{\circ}=1,799 \mathrm{Mm}$. (Mittel aus 8 Bestimmungen).

$x+\frac{r}{2}=10,365$. Radius im Scheitel $=7,74$ gesetzt.

Abstand des Drehpunktes vom Schẹitel $=x+r$ $=14,235$. Wenn auch die Differenz über $\frac{3}{10} \mathrm{Mm}$. beträgt gegenüber dem anderen Auge, so hatte ich bei Ausführung der Messungen doch die Ueberzeugung gewonnen, dass sich leicht die nöthige Fertigkeit im genauen Einstellen erlangen lässt. Ein sehr grosser Vortheil ist das bei weitem raschere Messen, umsomehr, da der Untersuchte häufig ungeduldig wird, wenn immer und immer wieder die Messung wiederholt werden muss. Bei den folgenden Bestimmungen habe ich daher vielfach diese leichtere Einstellung benutzt.

\section{Bestimmung.}

Herr stud. phil. v. W., 20 Jahr alt, aus Bern.

$\mathrm{H} \mathrm{m}=\frac{1}{40}$.

$\mathrm{S}$ mit freiem Auge binoculär $\frac{15}{12}$;

$$
\begin{array}{lll}
" \quad & \text { rechts } \cdot \frac{15}{12} ; \operatorname{mit}+40 \frac{15}{12} \\
" \quad & \text { links } . \frac{15}{15} ; \mathrm{mit}+50 \frac{15}{15} .
\end{array}
$$

binoculär No. 1 (J.) in $21^{\prime \prime}-4^{\prime \prime}$

rechtes Auge: No. 1 (J.) in $21^{\prime \prime}-4^{1 / 3^{\prime \prime}}$

linkes Auge: No. 1 (J.) in $20-4^{\prime \prime}$. 
Herr W., dessen Sehschärfe, wie aus den mitgetheilten Sehproben ersichtlich, eine sehr holie ist, klagt neben anderem über eine eigenthümliche Störung in der Farbenwahrnehmung. Diese näher zu untersuchen, schlug ich Herrn W. vor, der als Physiker Interesse an der Sache hatte, Spectralfarben mit dem Ophthalmometer zu mischen. Das Princip ist ungemein einfach. Das grösste Object, welches mit dem mir zur Verfügung stehenden Ophthalmometer gut gemessen werden kann, beträgt gegen $7 \mathrm{Mm}$. - Verschafft man sich nun ein etwa 6 bis $7 \mathrm{Mm}$. grosses Spectrum, was durch eine entsprechende Linse leicht $\mathbf{z u}$ bewerkstelligen ist, und betrachtet man dieses jetzt durch das Ophthalmometer, so sieht, man dasselbe einfach, wenn die Platten . parallel zu einander stehen, doppelt, wenn sie gegen einander gedreht werden. Durch Drehung der Platten lassen sich die 2 Spectren beliebig gegen einander verschieben und somit Theile des einen Spectrums mit Bestandtheilen des anderen zur Deckung bringen.

Statt des kleinen Spectrums lassen sich auch grössere verwenden. Durch passende Vorrichtungen muss man nur kleine Theile zweier grösserer Spectren so nahe zusammenbringen, dass man sie durch Drehung der Ophthalmometerplatten über einander verschieben kann. An anderer Stelle werde ich darauf zurückkommen*).

Winkel zwischen Hornhautaxe und Gesichtslinie $=5^{\circ}$. Breite der Basis corneae $=11,0 \mathrm{Mm}$. Verschiebung des Reflexbildes bei Drehung um $10^{\circ}=1,52 \mathrm{Mm}$. (Mittel aus 6 Bestimmungen. Grösste Differenz innerhalb der Reihe $=2,1^{\circ}$ ).

*) Ich hatte vorstehende Arbeit abgeschlossen, als ich von Herrn Prof. Nagel erfuhr, dass von Mandelstamm (Arch. f. Ophthal. XIII. 2. S. 399.) und später auch von Dobrowolsky ein ähnliches Verfahren in Helmholtz's Laboratorium angewendet worden ist, um den Grad der Empfindlichkeit der Netzhant für verschiedene Farbentone des Sonnenspectrums zu pruffen. 


$$
\begin{gathered}
\frac{172}{1,52} \\
\frac{\mathrm{x}+\frac{\mathrm{r}}{2}}{\mathrm{x}}=\sin 100 \\
\mathrm{x}+\frac{\mathrm{r}}{2}=8,7735 \mathrm{Mm} .
\end{gathered}
$$

Radius im Scheitel $=7,3 \mathrm{Mm}$. Abstand des Drehpunktes von dem Hornhautscheitel $=12,4235$.

An demselben Auge bestimmte ich jetzt nach dem von Donders und Doijer angewandten Princip den Drehpunktsabstand und fand 12,52. Um zu sehen, ob hierbei leichter und ausgiebigere Kopf bewegungen eintreten, gab ich der Bestimmung folgende Anordnung.

Befand sich das Auge in der Primärstellung, so wurde ein Licht in die verlängerte Hornhautaxe gebracht; auch das Spiegelbild lag dann auf der Hornhautaxe. Nun wurde das vertical gespannte Haar mittelst der Micrometerschraube vor das Reflexbild gebracht und ein Visirzeichen auf dem aus dem Drehpunkt beschriebenen Gradbogen so lange verschoben, bis das Haar gerade vor den Cornealrand za stehen kam. Wurde dann das Visirzeichen an die ursprüngliche Stelle zuriick gebracht, so musste, wenn keine Kopfbewegung stattgefunden hatte, das Haar das Reflexbild wieder decken. Dies war in der That unter einer Anzahl Bestimmungen auch mehrmals der Fall. Herr W. wusste, worauf es ankam und vermied auch bei stark seitlichem Blick die Mitbewegung des Kopfes. Anders verhält es sich im nãchsten Fall. Hier traten fast stets mit stark seitlicher Blickrichtung Bewegungen des Kopfes ein. Bei der Art und Weise, wie Donders und Doijer die Bestimmung ausführen, wird allerdings der Einfluss von Kopf bewegungen einigermassen verhütet. 
IV. Bestimmung.

Herr S., Küfer, 30 Jahr alt, aus Gommeringen.

Linkes Auge: mit freiem Auge $\mathrm{S}=\frac{15}{15} ; \mathrm{mit}+80 \frac{15}{15}$.

Rechtes Auge: (traumat. Mydriasis) mit freiem Ange

$$
\mathrm{S}=\frac{15}{20} ; \mathrm{mit}+80 \frac{15}{20}
$$

Rechtes Auge: No. 1 (J.) in 38 bis $16 \mathrm{~cm}$.

Linkes Auge: No. $1\left(J_{2}\right)$ in 49 bis $10 \mathrm{~cm}$.

Nahepunkt mit Fadenoptometer bestimmt.

Rechtes Auge: $\left.16 \frac{1}{2} \mathrm{Cm} .{ }^{*}\right)$.

Linkes Auge: $10 \% \mathrm{Cm}$.

Rechtes Auge: Winkel zwischen Hornhautaxe und Gesichtslinie $=5^{1 / 2^{0}}$. Durchmesser der Basis corneae $=10,9 \mathrm{Mm}$. Bei Drehung des Auges um $10^{\circ}$ aus der Primärstellung erleidet das Reflexbild eine Verschiebung von $1,59 \mathrm{Mm}$. (Drehung der Platten des Ophthalmometers $=23^{\circ}, 17$. Mittel aus 5 Messungen. Grösste Differenz innerhalb der Reibe der beobachteten Drehungswerthe $=1,2^{0}$.)

$$
x+\frac{r}{2}=9,35
$$

Radius im Hornhautscheitel $=7,5$. Abstand des Drehpunktes von dem Hornhautscheitel $=13,1 \mathrm{Mm}$.

Nach der Donders-Doijer'schen Methode wurde $12,849 \mathrm{Mm}$. erhalten.

\section{Bestimmung.}

Eduard N., 16 Jahr alt, Gärtner aus Göttelfingen.

Linkes Auge: mit freiem Auge $S=\frac{15}{15}$; mit $+36 \frac{15}{15}$.

*) Auf mehrmaliges Einträufeln von Calabar wird am rechten Auge Pupille enger und Nahepunkt rückt anf $13 \% / 2 \mathrm{Cm}$. heran. 
Rechtes Auge: mit freiem Auge $S=\frac{15}{70} ;$ mit $+18 \frac{15}{40^{\circ}}$.

Stärkere Convexgläser werden von dem rechten Auge nicht angenommen. Mit Cylindergläsern ist eine Verbesserung bis zu $\frac{15}{20}$ ? zu erzielen.

Linkes Auge. Pupillendistanz $=2^{\prime \prime} 4^{\prime \prime \prime}$ (Wiener Maass). Winkel zwischen Hornhautaxe und Gesichtslinie $=51 \%^{\circ}$. Durchmesser der Hornhautbasis $=11,2 \mathrm{Mm}$. Verschiebung des Refexbildes bei einer Drehung des Auges um $10^{\circ}=1,53 \mathrm{Mm}$. (Drehung der Ophthalmometerplatten im Mittel $=22,41^{\circ}$. Mittel aus 5 Messungen. Grösste Differenz der Reihe $=1,9^{\circ}$.)

$$
x+\frac{r}{2}=8,8 \text {. }
$$

Radius im Hornhautscheitel $=7,2 \mathrm{Mm}$. Abstand des Drehpunktes von dem Hornhautscheitel $=12,4 \mathrm{Mm}$.

\section{Bestimmung.}

Herr L., stud. phil., 20 Jahr alt.

Linkes Auge: (atropinisirt) M $\frac{1}{5}$. Măssig grosse Sichel nach aussen von der Papille.

Winkel zwischen Hornhautaxe und Gesichtslinie $=24^{\circ}$. Durchmesser der Hornhautbasis $=11,4 \mathrm{Mm}$. Krümmungsradius im Hornhautscheitel $=7,64$. Verschiebung des Reflexbildes der Cornea, wenn das Auge eine Drehung um $10^{\circ}$ ausführte $=1,75$ (Mittel aus $10 \mathrm{Ab}$ lesungen. Grösste Differenz innerhalb der Reihe $2,2^{0}$ ).

$$
x+\frac{r}{2}=10,077
$$

Abstand des Drehpunktes hinter dem Hornhautscheitel $=13,897$. 
VII. Bestimmurg.

Georg G.*), Gymnasiast, $17 \mathrm{Jahr}$ alt, aus Tübingen.

Rechtes Auge. Pupillendistan $z=2^{\prime \prime} 5^{\prime \prime}$. Winkel zwischen Hornhautaxe und Gesichtslinie $=5^{1 / 2}$. Durchmesser der Hornhautbasis $=11,64 \mathrm{Mm}$. Radius im Scheitel $=7,6 \mathrm{Mm}$.

Verschiebung des Reflexbildes der Cornea, wenn das Auge eine Drehung um $10^{\circ}$ ausführt $=1,58 \mathrm{Mm}$. (Drehung der Ophthalmometerplatten $=23,0^{\circ}$, Mittel aus 5 Bestimmungen. Grösste Differenz innerhalb der Reihe $0,7^{\circ}$ )

$$
x+\frac{r}{2}=9,099
$$

Lage des Drehpunktes hinter dem Hornhautscheitel $12,899$.

VIII. und IX. Bestimmung.

Herr S., stud. phil., 22 Jahr, aus England.

$\mathrm{S}$ binocul. $\frac{15}{15}$.

$S$ rechtes Auge: mit freiem Auge $\frac{15}{15}$.

$\mathrm{S}$ linkes Auge: mit freiem Auge $\frac{15}{30^{\circ}}$

Convexgläser werden nicht angenommen, an dem linken Auge bessert ein schwaches Concavglas bis $\mathrm{zu}$ $\frac{15}{15}$. Mit dem Augenspiegel beiderseits hypermetrop. Einstellung bestimmt. Geringer Astigmatismus. HerrS. hat viel mit feinem Druck zu arbeiten, besonders hebräischer Schrift. Als er später, nachdem er mehrere Tage hinter

*) An anderer Stelle ist dieser Fall von scheinbarer Myopie năher mitgetheilt. 
einander angestrengt mit solchem geal beitet hatte, sich wieder vorstellt, liest er mit dem rechten Auge nur $\frac{15}{20}$, mit dem linken Auge $\frac{15}{30} ? ; \frac{15}{15}$ erhält er erst mit -40 am rechten Auge, $\frac{15}{20}$ und einzelne Buchstaben von $15 \mathrm{mit}$ - 50 am linken Auge. Mit dem Augenspiegel wie früher hypermetrop. Einstellung.

Eine vorgeschlagene Atropinkur durchzumachen, erlaubt augenblicklich Herrn S. seine Zeit nicht.

Linkes Auge: Winkel zwischen Hornhautaxe und Gesichtslinie $=4^{\circ}$. Durchmesser der Hornhautbasis $=11,15$. Verschiebung des Cornealreflexes, wenn das Auge um $10^{\circ}$ gedreht wird $=1,61 \mathrm{Mm}$. (Drehung der Ophthalmometerplatten $=23,333^{\circ}$, Mittel aus 5 Bestimmungen. Grösste Differenz innerhalb der Reihe $=1,8^{\circ}$ ). Radius im Hornhautscheitel $=7,4 \mathrm{Mm}$.

$$
\mathrm{x}+\frac{\mathrm{r}}{2}=9,2716 \text {. }
$$

Abstand des Drehpunktes vom Hornhautscheitel $=12,97$.

Rechtes Auge: Winkel zwischen Hornhantaxe und Gesichtslinie $=41^{\circ}$. Verschiebung des Cornealreflexbildes bei Drehung des Auges um $10^{\circ}=1,62 \mathrm{Mm}$. (Mittel aus 4 Bestimmungen. Grösste Differenz innerhalb der Reihe $0,6^{\circ}$ ).

$$
\mathrm{x}+\frac{\mathrm{r}}{2}=9,3292 \text {. }
$$

Abstand des Drehpunkts vom Hornhautscheitel $=13,03 \mathrm{Mm}$.

\section{Bestimmung.}

Herr F., Lehrer, 32 Jahr alt, aus Münzdorf, erhielt vor mehreren Jahren von einem Optiker ein Concav- 
glas 9, mit dem Rath, dasselbe sowohl zum Sehen in die Ferne, als auch zum Sehen in die Nähe stets zu tragen. Lange Zeit war Herrn F. dieses (für seine Myopie zu starke) Glas angenehm, bis er im Mai dieses Jahres (1874) bemerkte, dass er mit dem rechten Auge (mit vorgesetztem Glas) nicht mehr so gut lesen konnte wie früher. Ende August machte er die gleiche Wahrneh. mung am linken Auge und dieses Schlechtsehen in der Nähe bei vorgesetztem Concavglas nahm zu. Weiter giebt Herr F., der sich ängstlich beobachtet zu haben scheint, an, im Mai habe er im Spiegel eine Erweiterung der Pupille des rechten, beziehungsweise später im August des linken Auges bemerkt.

Bei der am 24. November 1874 vorgenommenen Sehprüfung sieht er

linkes Auge: mit freiem Auge $S=\frac{15}{200} ;$ mit $-11 \frac{15}{20}$, selbst einzelne Buchstaben von 15 ,

rechtes Ange: mit freiem Ange $S=\frac{15}{200} ;$ mit $-11 \frac{15}{20}$ bis $\frac{15}{15}$ ?

binoc. No. 1 (J.) in $13^{\prime \prime}-5^{1 / 2}{ }^{\prime \prime}$

rechtes Auge: No. 1 (J.) in $12^{1} 4^{11}-5^{3} / 4^{11}$

linkes Auge: No. 1 (J.) in $12^{3} /_{4}{ }^{\prime \prime}-6^{3} /_{4}{ }^{\prime \prime}$.

Als die Sehstörung im Mai anfing, hatte er keine Schmerzen. Solche kamen erst später - besonders in der Nacht - und hatten ihren Sitz in der Stirn. Für das Auftreten der Schmerzen soll ohne Bedeutung gewesen sein, ob Herr F. sich lange anhaltend mit Arbeit in der Nähe beschäftigt hatte. Ohne Brille sah er dem Grad seiner Myopie entsprechend in der Nähe vollständig gut. Er entschloss sich aber nicht - aus unverständlichen Gründen - beim Sehen in die Nähe die Brille abzulegen.

Links reagirt die Pupille träge, rechts bleibt sie auf Lichteinfall unbeweglich.

₹. Graefe's Archiv für Ophthalmologie, XXI. 2. 
Mit dem Augenspiegel untersucht, ergeben sich die Medien rein. Refraction vielleicht etwas geringer myopisch, als es dem Grad; der durch Gläser gefunden wurde, entspricht. Grenzen der Papille verwischt, namentlich nach innen. An dieser Stelle sind die Gefässe leicht geschlängelt. An die Papille schliesst sich keine Sichel an.

Aus der Anamnese wäre noch einer syphilit. Ansteckung vor 4 Jahren za erwähnen. Nach einem ulcus penis sollen bald darauf Drüsen angeschwollen sein und etwas später auch ein fleckiger Ausschlag auf der Brust sich gezeigt haben. Später habe sich niemals mehr eine als syphilitisch zu deutende Affection gezeigt.

Gegen die Mydriasis wurde Electricität und Calabar ohne Erfolg versucht.

Wurde das Verhalten der Pupille am rechten Auge näher untersucht, so zeigte sich Folgendes: Auf Lichteinfall, selbst auf das durch ein Convexglas gesammelte, bleibt die Pupille vollständig unverändert; bei accommodativer Anstrengung erfolgt eine geringe Verengerung; eine sehr auffallende Verengerung tritt constant bei Bewegungen des Auges nach aussen ein. Um bei der Bewegung nach aussen eine accommodative Anstrengung auszuschliessen, wird ein in grosser Entfernung vor dem Auge befindliches Object seitlich verschoben und das Ange angewiesen, demselben mit dem Blick zu folgen*). Gesichtsfeld normal. Farbenempfindung gut.

*) Eine abnorme Verbindung zwischen Abducens und Oculomotorius ist von verschiedenen Seiten anatomisch nachgewiesen Merkel im Graefe-Sämisch'schen Handbuch I. 1, S. 129). Vielleicht besteht in vorliegendem Fall eine derartige abnorme Verbindang. Eine accommodative Anstrengung war mit der bei Bewegung nach aussen eintretenden Pupillenverengerung nicht verbunden. 
Es besteht geringe Insufficienz der Interni.

Es werden versuchsweise Strychnininjectionen gemacht, um zu sehen, ob damit eine Reaction in der Pupille erfolgt -, doch ohne Erfolg.

Nachdem die Augen atropinisirt sind, wird mit freiem Auge rechts $\left.\begin{array}{c}15 \\ \text { links }\end{array}\right\} \frac{15}{200}$

gesehen.

Rechtes Auge: mit $-12 \mathrm{~S}=\frac{15}{15}$; combin. mit Convexcylinder 36, Axe horizontal, Buchstaben von 12.

Linkes Auge: mit $-11 \mathrm{~S}=\frac{15}{15}$; combin. mit Convexcylinder 30, Axe horizontal, Buchstaben von 12. Die Pupillendistanz ist etwas grösser als gewöhnlich.

Die Augen zeigen nicht das gewöhnliche Aussehen myopischer Augen. Die Bulbi scheinen sehr klein; bei seitlicher Blickrichtung fällt die rasch abfallende Krümmung nach hinten auf. Die vordere Kammer ist eng.

Rechtes Auge: Winkel zwischen Hornhautaxe und Gesichtslinie $=21^{1 / 4^{0}}$. Durchmesser der Hornhautbasis $=9,9 . \mathrm{Mm}$. Krümmungsradius im Scheitel $=6,76 \mathrm{Mm} .{ }^{*}$ )

Im III. Bd, 74.Abth., S. 363 dies. Archivs theilt v. Graefe Beobachtungen mit uber bei Bewegung nach aussen eintretende Pupillenverengerung an Augen, deren Oculomotorius gelähmt ist. Er hält es für möglich, dass in solchen Fällen bei erhaltener peripherer Reizbarkeit der Oculomotoriusfasern durch die Rotation des Bulbus (bei Contraction des normal fungirenden Abducens) mechanisch ein Contractionsimpuls bedingt werde. Mit einer solchen Deutung in Einklang stehend sah $\nabla$. Graefe in einem jener Fälle bei Contraction des Trochlearis gleichfalls eine Pupillenverengerung angedeutet.

*) Am folgenden Tage wurden der Controle wegen die Bestimmungen wiederholt. Als Mittel aus 6 Bestimmungen wurde fur den Krümmungsradius 6,72 $\mathrm{Mm}$. gefunden. 
(Mittel aus 4 Bestimmungen). Verschiebung des Reflexbildes der Cornea bei einer Drehung des Auges um $8^{\circ}$ $=1,25 \mathrm{Mm}$.

Aus der Grösse. dieser Verschiebung bei bekanntem Krümmungsradius berechnet sich der Abstand des Drehpunktes hinter Hornhautscheitel $=12,36 \mathrm{Mm}$.

Vor allem muss hier der kleine Krümmungsradius der Hornhaut auffallen*). Nimmt man die enge vordere Kammer hinzu und die Kleinheit des Bulbus im Ganzen, so dürfte man wohl berechtigt sein, den vorliegenden Fall von Myopie als eine Myopie bedingt durch stärkere Krümmung anzusehen. Dafür spricht auch die Lage des Drehpunktes.

Der Uebersichtlichkeit halber sind die mitgetheilten 10 Drehpunktsbestimmungen in folgender Tabelle zusammengestellt (s. S. 181).

Ich füge hier noch 2 Drehpunktsbestimmungen an, welche in den letzten Tagen an 2 hochgradig myopischen Augen mit beschränkter Beweglichkeit ausgeführt wurden. Der Drehpunkt lag in einem Fall $\left(\mathrm{M} \frac{1}{2^{1 / 2}}\right)$ 14,241 Mm., im anderen $\left(\mathrm{M} \frac{1}{5^{1 / 2}}\right) 14,8 \mathrm{Mm}$. hinter dem Hornhautscheitel.

Vergleicht man die in der Tabelle enthaltenen Werthe mit den von Donders angegebenen Mittelwerthen, so erscheinen die gefundenen Werthe im Ganzen etwas kleiner. Bei der kleinen Zahl der Bestimmungen ist daraus nichts zu folgern, um so weniger, als innerhalb der Don ders'schen Bestimmungsreihen für dieselbe

*) Wird der Brechungsindex der Cornea = 1,3365 gesetzt, so erbălt man für diese Cornea eine Brennweite in Luft $=20,08$, eine Brennweite in Humor aqueus $=26,91$, während im schematischen Auge die vordere Brennweite $=23,69$, die hintere $=31,69$ gesetzt ist. (Donders, die Anomalien der Refraction und Accommodation, S. 58), 


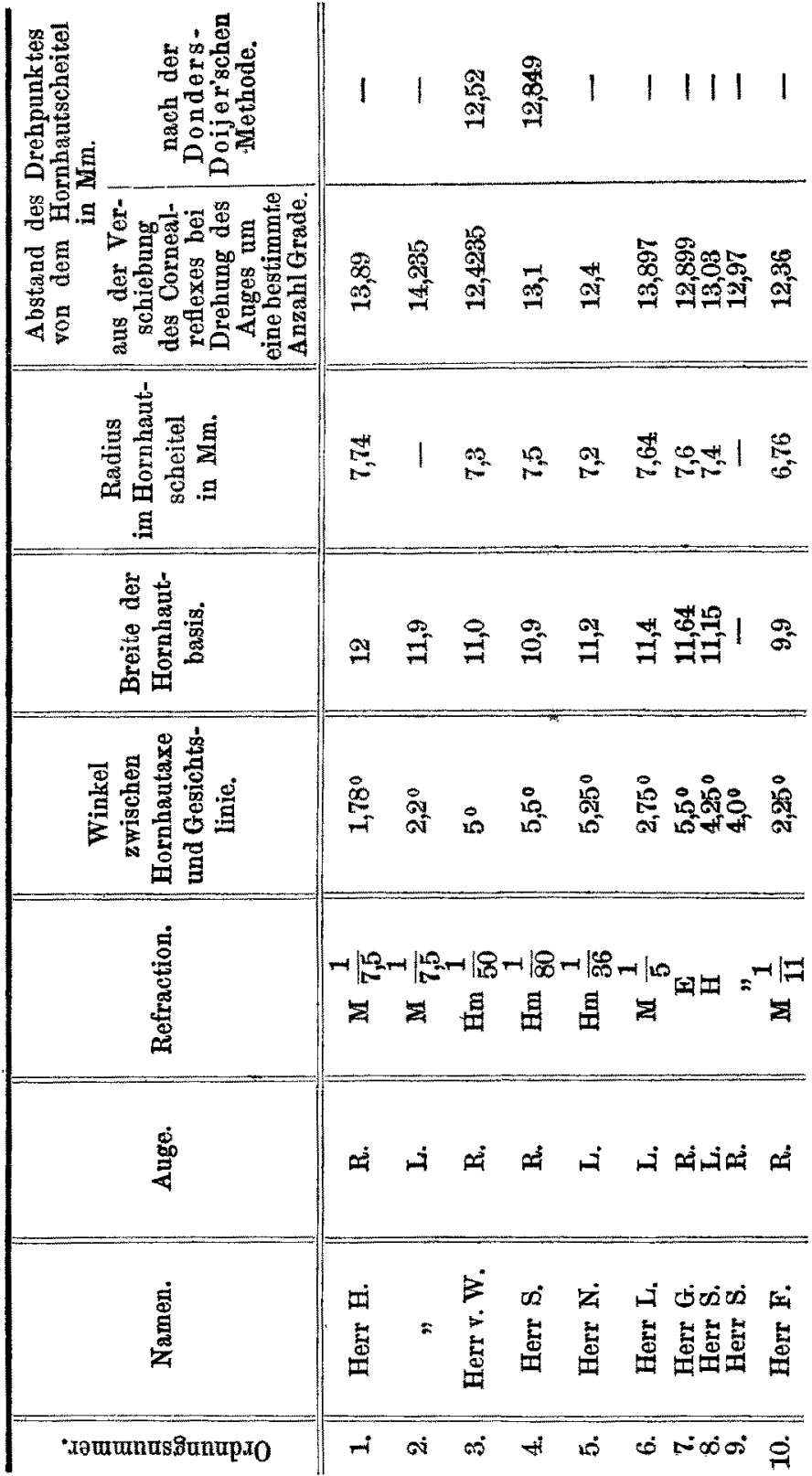


Refractionsanomalie ziemlich grosse Differenzen sich zeigen.

Als Mittelwerthe giebt Donders an:

für das emmetropische Auge: . 13,45 $\mathrm{Mm}$.

" " myopische Auge: . . . 14,52 $\mathrm{Mm}$.

$"$ " hypermetropische Auge: 13,22 $\mathrm{Mm}$.

Innerhalb der Reihe der Bestimmungen für 15 emmetropische Augen beträgt die grösste Differenz 1,01 Mm., für 12 hypermetropische Augen 2,35 und für 17 myopische 2,89 $\mathrm{Mm}$. Diese grossen Differenzen innerhalb der Reihen für Ametropie erklären sich hauptsächlich daraus, dass die Lage des Drehpunktes im Grossen und Ganzen mit dem Grad der Refractionsanomalie zusammenhängt, so dass der Drehpunkt im hochgradig myopischen Auge im Allgemeinen weiter nach hinten liegt, dem entsprechend, dass bekanntlich die Myopie in den meisten Fällen auf Axenverlängerung beruht. Zum anderen Theil hängen aber diese Differenzen davon $a b$, $o b$ man es mit einem durch Axenverlängerung oder durch stärkere Krümmung myopischen Auge zu thun hat. In der Reihe von Donders und Doijer befinden sich zwei Augen mit Myopie $\frac{1}{6^{1 / 4}}$. Als Abstand des Drehpunktes von der Hornhautbasis wurde bei dem einen 10,58 , für denselben Abstand am anderen 12,37 gefunden*). Es liegt nahe, hier wie auch in Bestimmung 10 an die erwähnte Verschiedenheit zu denken.

Zum Schluss nehme ich Gelegenheit meinem geehrten Lehrer, Herrn Prof. C. Eckhard in Giessen, für die Freundlichkeit, womit er mir sein Ophthalmometer geliehen hat - sowie auch Herrn Prof. Nagel für die Er-

*) Arch. für holländ. Beiträge. Bd. III. S. 271. 
laubniss der Benutzung der Krankenprotokolle meinen besten Dank auszusprechen.

Wenn auch nicht direct zum Gegenstand gehörend, so doch eng mit ihm zusammenhängend ist die Verwendung des Cornealreflexes zur Entscheidung über den Sitz einer Trübung im Auge. Um so lieber gehe ich mit wenigen Worten auf diesen Gegenstand ein, als ich von ihm bei der vorliegenden Arbeit ausgegangen bin.

In dem vortrefflichen Lehrbuch der Ophthalmoskopie von Mauthner heisst es hierüber (S. 155): „bei dem Beginn des Versuchs liegen Hornhautreflex und Drehpunkt in der Verlängerung der Gesichtslinie des Beobachters. Geht das Auge aus der ursprünglichen Stellung heraus, so bleiben 2 Punkte im Raume unbeweglich, bleiben auf derselben Linie liegen, der Hornhautreflex und der Drehpunkt nämlich. Eine Trübung, die im Drehpunkt ihren Sitz hat, bleibt bei allen Bewegungen des Auges vom Hornhautreflex gedeckt. Alle übrigen Opacitäten weichen hierbei zur Seite und zwar entfernen sich die vor dem Drehpunkt gelegenen vom Hornhautreflex nach jener Richtung, nach der das Auge geht, die hinter dem Drehpunkt lagernden dagegen nach der entgegengesetzten Seite."

Wenn G G (siehe Figur 11 auf der folgenden Seite) die Gesichtslinie des Beobachters, dessen Auge sich in 0 befindet, darstellt und in $L$ das Bild der in $\mathbf{F}$ stehenden Flamme liegt und zwar, wenn S S ein Planspiegel ist, ebenso weit hinter dem Spiegel, wie $F$ vor dem Spiegel - dann wird das Cornealbild 1 mit dem Drehpunkt $D$ dann gleichzeitig in der Gesichtslinie G G liegen, wenn die Hornhautachse des untersuchten Auges in die Gesichtslinie GG des Beobachters fällt. 
Fig. 11.

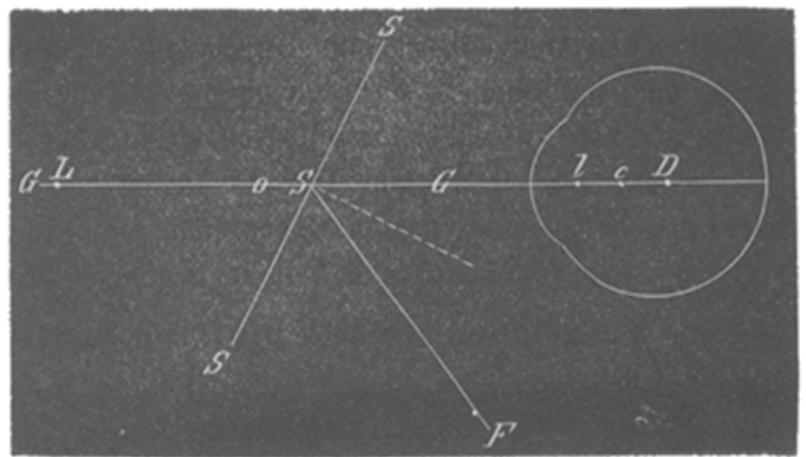

Führt das Auge des Untersuchten eine Bewegung aus bei sonst gleich bleibenden Verhältnissen, so wird. nur der Punkt $D$ auf der Linie $G$ G bleiben, der Krümmungsmittelpunkt der sphärisch angenommenen Cornea wird sich im Sinn der Drehung als vor dem Drehpunkt liegend nach $c^{\prime}$ (Fig. 12) bewegen. Das Reflex-

Fig. 12.

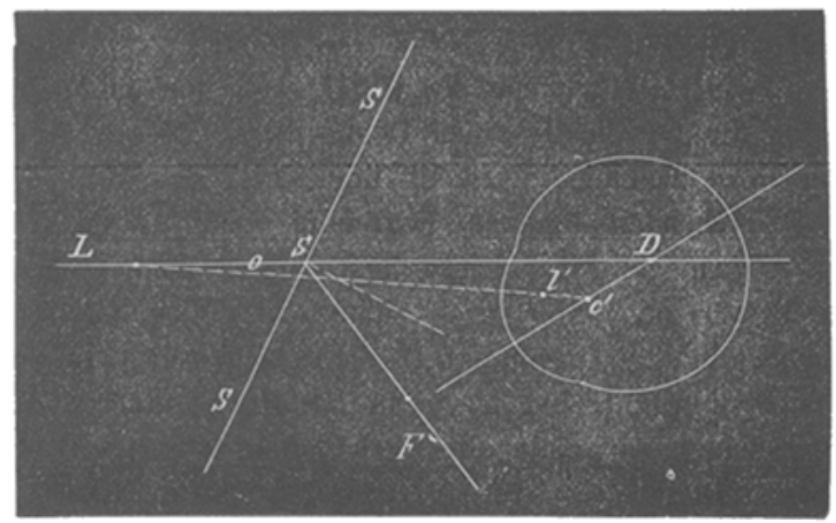


bild liegt jetzt auf der Verbindungslinie von $\mathrm{L}$ mit $\mathrm{c}^{t}$ etwa in 1 .

Eine Opacität im Drehpunkt wird jetzt nicht mehr von dem Reflexbild gedeckt sein, wohl aber wird dieses der Fall sein für eine Trübung, die an der Stelle des Krümmungsmittelpunktes der Cornea ihren Sitz hat.

Hierbei ist aber zweierlei zu bemerken, erstens dass die Cornea sphärisch angenommen ist bei der vorstehenden Betrachtung und dann - dass unter dieser Annahme die Betrachtung absolut genau richtig nur dann ist, wenn das Auge des Beobachters sich in $\mathrm{L}$ oder doch wenigstens sehr nahe bei $\mathrm{L}$ befindet.

Das eben Gesagte dürfte in Betracht kommen für das, was Mauthner (l. c., S. 156) weiter unten angiebt. "Der hintere Kapselstaar wird von dem Hornhautreflex bei Bewegungen des Auges nicht gedeckt bleiben können. Ist dies letztere der Fall, wie es nicht selten in Fällen von Chorioiditis und Retinitis pigmentosa bei derartigen Trübungen, welche in der Nähe des hinteren Linsenpols' zu liegen scheinen, geschieht, so sind wir genöthigt, den Sitz derselben in den Glaskörper zu verlegen."

Nach der obigen Auseinandersetzung ist hierzu kein zwingender Grund vorhanden.

Sobald ich mir über die hier in Betracht kommenden Verhäitnisse klar war, erkannte jch die Möglichkeit, aus der Verschiebung des Reflexes der Cornea bei einer bestimmten Drehung des Auges die Lage des Drehpunktes zu ermitteln. In Weiterverfolgung biervon fand ich von Junge dieses Princip verwendet 
- auch von Donders (selbstständig ohne Kenntniss der Junge'schen Arbeit) angewandt, aber wieder verlassen wegen des einen Fehler bedingenden Einflusses, den die Ellipticität der Cornea auf die Verschiebung ausübt. Wie gezeigt, lässt sich aber der letztere leicht vermeiden.

Tübingen, Dezember 1874. 